
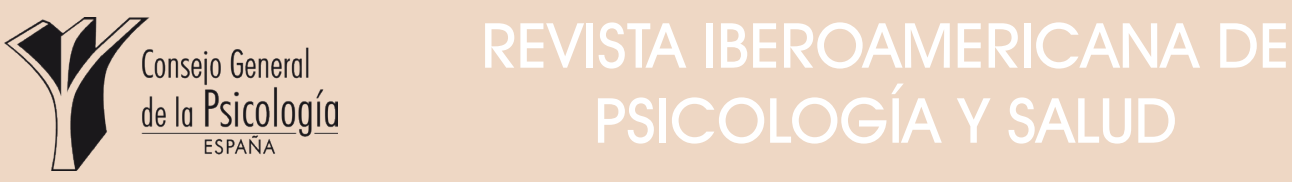

Revista Oficial de la Federación Iberoamericana de Asociaciones de Psicología (FIAP)

[Official Journal of the Latin-American Federation of Psychological Associations]

\title{
The role of psychopathic traits among intimate partner-violent men: A systematic review
}

\author{
Asunción Fernández-Suárez", Beatriz Pérez², Juan Herrero', Joel Juarros- \\ Basterretxea $^{1}$ \& Francisco Javier Rodríguez-Díaz'
}

'Departamento de Psicología. Universidad de Oviedo, ${ }^{2}$ Núcleo en Ciencias Sociales y Humanidades. Universidad de la Frontera

- Received: 08 - 01 - 2018 - Aceppted: 07 - 05 - 2018 . Avaliable online: 17 - 05 - 2018

ABSTRACT: Preventing intimate partner violence (IPV) requires effective batterer intervention programs. Although psychopathy seems to be a key variable for successful treatment, little is known about the role of psychopathic traits among male IPV perpetrators. Therefore, a systematic review of all empirical quantitative studies $(N=41)$ on the relationship between psychopathic traits and male-to-female IPV perpetration was carried out. Overall, the findings from these studies supported the role of psychopathy as a robust predictor of male-tofemale IPV perpetration among both convicted and non-convicted partner-violent men. Male batterers do not seem to be a homogeneous group in terms of their psychopathic traits. The existence of a batterer subtype consistent with successful psychopathy and its implications for treatment outcomes should be further examined. Although psychopathy seems to be a useful variable to predict frequency of IPV (and then, IPV recidivism), this construct seems to fail to predict IPV severity. Further research is necessary to clarify the role of psychopathic traits on IPV risk assessment. Methodological deficiencies, mainly related to psychopathy measurement, have been evidenced. Alternative psychopathy assessment tools that exclude criminality/antisociality as diagnostic criteria are recommended.

KEYWORDS: Intimate partner violence, Batterer, Psychopathy, Psychopathic traits, Systematic review.

\section{El rol de los rasgos psicopáticos entre agresores de pareja: Una revisión sistemática}

RESUMEN: La prevención de la violencia en la pareja (IPV) exige un tratamiento efectivo de los agresores. Aunque la psicopatía parece ser una variable clave para el éxito del tratamiento, poco se sabe sobre el rol de la psicopatía entre agresores de pareja. Se ha realizado una revisión sistemática de artículos empíricos cuantitativos $(N=41)$ sobre la relación entre psicopatía y la comisión de IPV. En general, los resultados apoyaron el rol de la psicopatía como un fuerte predictor de IPV, tanto entre agresores de pareja condenados como no condenados. Los agresores de pareja no parecen ser un grupo homogéneo en términos de psicopatía. La existencia de un subtipo de agresor consistente con el psicópata de éxito y sus implicaciones para el tratamiento deberían ser estudiadas en profundidad. Aunque la psicopatía parece ser una variable útil para predecir la frecuencia de IPV, este constructo parece fallar a la hora de predecir la gravedad de IPV. Se necesita más investigación para clarificar el rol de la psicopatía en la evaluación del riesgo de IPV. Se han detectado deficiencias metodológicas, principalmente relacionadas con la medición de la psicopatía. Se recomiendan instrumentos de evaluación alternativos, los cuales excluyan la criminalidad/antisocialidad como criterio diagnóstico.

PALABRAS CLAVE: Violencia en la pareja, Agresor de pareja, Psicopatía, Rasgos psicopáticos, Revisión sistemática.

Preventing intimate partner violence (IPV) requires coordinated actions in the criminal justice and social service response systems, such as services and advocacy programs for victims and effective batterer intervention programs

\footnotetext{
*Correspondence: Asunción Fernández Suárez.

Departamento de Psicología. Universidad de Oviedo.

Postcode: 33003. Oviedo, España.

E-mail: asunfdezsuarez@gmail.com
}

(BIPs; Aaron \& Beaulaurier, 2016). Previous meta-analysis about the efficacy of BIPs suggest a minimal impact of BIP on reducing recidivism (Arias, Arce, \& Vilariño, 2013; Babcock, Green, \& Robie, 2004; Feder \& Wilson, 2005). BIPs' effectiveness depends not only on the context of the intervention but also on both the therapist and offenders' characteristics (Carbajosa, Boira, \& Tomás-Aragonés, 2013; Carbajosa, Catalá-Miñana, Lila, \& Gracia, 2017; Sjödin, Wallinius, Billstedt, Hofvander, \& Nilsson, 
2017). The literature suggests that the offenders' characteristics as psychopathic traits are a key variable for successful treatment (Richards et al., 2016). Psychopathic individuals show less motivation to change and are likely to deceive and manipulate the therapist, to have problems during treatment and to drop out of treatment (Richards et al., 2016; Salekin, Worley, \& Grimes, 2010). These negative treatment outcomes lead to therapeutic pessimism; however, the belief that the psychopath is untreatable seems to have little scientific support (Salekin, 2002). It is likely that conventional intervention strategies are ineffective to treat psychopaths because they learn better techniques to manipulate others (Moreira, Almeida, Pinto, \& Fávero, 2014). Instead, some authors suspect that specific interventions that take psychopathic traits into consideration might have a positive impact on treatment outcomes (Felthous, 2015; Reidy, Kearns, \& DeGue, 2013); recent studies implementing alternative and specific intervention programs targeted at psychopathic individuals have obtained encouraging results (Burt, Olver, \& Wong, 2016; Dargis, Mattern, \& Newman, 2017). Thus, research targeted at the identification of psychopathic batterers is imperative to design effective, specific BIPs for psychopathic batterers.

Psychopathy remains a controversial construct (Scott, 2014): there is still disagreement today over the essential or "core" psychopathic traits (Miller, Lamkin, Maples-Keller, \& Lynam, 2016). This construct has been commonly defined as a personality disorder characterized by a cluster of interpersonal, affective, and lifestyle traits. However, while some authors consider that antisociality/criminality is a fundamental component of the psychopathy construct (Hare, 2016), other contemporary researchers (Skeem \& Cooke, 2010) note that antisocial traits are a mere correlate or consequence of psychopathy and thus should not be included as diagnostic criteria to assess psychopathy.

The conceptual confusion surrounding psychopathy has raised a debate about whether antisocial traits are necessary diagnostic criteria in psychopathy assessment. The Psychopathy Checklist-Revised (PCL-R; Hare, 2003), the international psychopathy assessment tool most commonly used in both adult forensic and clinical samples (Hare, 2016), includes the measurement of antisocial features. Based on
PCL-R, other scales such as the Psychopathy Checklist: Screening Version (PCL-SV; Hart, Cox, \& Hare, 1995) or the Self-Report of Psychopathy (SRP; Paulhus, Neumann, \& Hare, 2016) have been developed. PCL-R and PCL-SV application requires the combination of information from semi-structured interviews and file records (Hare, 2003; Hart et al., 1995). Unlike Hare's model of psychopathy, alternative psychopathy measurements have been proposed that exclude criminal/antisocial behavior as diagnostic criteria and are thus able to differentiate psychopathic from antisocial traits (Evans \& Tully, 2016). The Psychopathy Personality InventoryRevised (PPI-R; Lilienfeld \&Widows, 2005) or the alternative 13-item PCL-R based on the 3-factor hierarchical model (Cooke, Michie, \& Skeem, 2007; Pérez, Herrero, Velasco, \& RodríguezDíaz, 2015) are two of these instruments. Hare's PCL-R and alternative psychopathy measures seem to assess the same underlying construct regardless of they include antisocial or criminal behavior as diagnostic criteria (Salvador, Arce, Rodríguez-Díaz, \& Seijo, 2017). Regardless of the model employed to assess psychopathic traits, this construct can be measured from both a categorical and dimensional approach.

Although there have been theoretical studies examining the role of psychopathic traits in partnerviolent men (Huss, Covell, \& LanghinrichsenRohling, 2006; Huss \& Langhinrichsen-Rohling, 2000; Juodis, Starzomski, Porter, \& Woodworth, $2014 b)$, to date, there has been no systematic review that uses explicit methods to identify, select, and critically appraise relevant research and to collect and analyze data from this research (Moher, Liberati, Tetzlaff, Altman, \& The PRISMA Group, 2009). Despite a large body of literature suggesting the strong predictive power of psychopathy on dangerousnessviolent and aggressive behavior (Hecht, Berg, Lilienfeld, \& Latzman, 2016; Reidy et al., 2015), violent criminal behavior (Dil \& Kazmi, 2016; Wiklund, Ruchkin, Koposov, \& Af Klinteberg, 2014), recidivism (Richards et al., 2016; Sturup, Karlberg, Fredriksson, Lihoff, \& Kristiansson, 2016), and even violent recidivism (Richards et al., 2016; Sitney, Caldwell, \& Caldwell, 2016)_little is known about the role of psychopathic traits in IPV perpetration. Although traditional batterer typologies have suggested that psychopaths could belong to a specific batterer subtype-type 
1 batterers, proposed by Gottman et al. (1995), and Jacobson and Gottman (1998), or generally violent/antisocial (GVA) batterers, proposed by Holtzworth-Munroe and Stuart (1994)_further empirical studies testing these hypotheses are necessary.

A systematic review of the role of psychopathic traits in male-to-female IPV perpetration would help to (a) highlight future directions for research about batterer typologies, (b) underscore the need to take into account psychopathy traits for risk management, and (c) provide recommendations for designing specific BIPs for psychopathic batterers. The aim of this systematic review is to examine the role of psychopathic traits to predict male-to-female IPV perpetration and to differentiate among male batterer subtypes, paying special attention to the type of samples analyzed and the assessment tools used to measure psychopathy.

\section{METHOD}

A systematic review was carried out in accordance with PRISMA guidelines (Moher et al., 2009) in order to identify peer-reviewed papers that empirically examined the role of psychopathic traits in male-to-female IPV perpetration. For inclusion in this review, no constraints were placed on the publication date (the only limiting factor was the starting points of the databases themselves) or language. However, restrictions were put in place as to document type, limiting the search to empirical quantitative articles. For a full list of inclusion and exclusion criteria for identification of relevant literature in this review, see Table 1.

\section{-SEARCH STRATEGY AND ARTICLE SELECTION}

Literature searches were performed in three different bibliographic databases (PsycINFO, Scopus, and Web of Science [WoS: All databases, specifically WoS Core Collection, Current Contents Connect, Derwent Innovations Index, $\mathrm{KCl}$ Korean Journal Database, MEDLINE, Russian Science Citation Index, and SciELO Citation Index]). The following combination of keywords (always in quotation marks) was entered into the different databases: "Psychopathy" OR "Psychopathic") AND ("Intimate Partner Violence" OR "IPV" OR "Partner abuse" OR "Male

\begin{tabular}{|c|c|}
\hline $\begin{array}{r}T a \\
\text { Inclusion and }\end{array}$ & $\begin{array}{l}\text { le } 1 \\
\text { exclusion criteria }\end{array}$ \\
\hline Studies included (were) & Studies excluded (were) \\
\hline \multirow{6}{*}{$\begin{array}{l}\text { Empirical quantitative } \\
\text { studies }\end{array}$} & - Book chapters \\
\hline & -Phd Theses/dissertations \\
\hline & -Editorials \\
\hline & - Congress abstracts \\
\hline & $\begin{array}{c}\text {-Theoretical and meta- } \\
\text { analytic studies }\end{array}$ \\
\hline & $\begin{array}{l}\text {-Empirical qualitative } \\
\text { studies }\end{array}$ \\
\hline \multirow{4}{*}{$\begin{array}{l}\text { Conducted with adult } \\
\text { men violent against their } \\
\text { female partners or studies } \\
\text { which assessed male-to- } \\
\text { female IPV in adulthood }\end{array}$} & $\begin{array}{l}\cdot \text { Conducted with } \\
\text { experienced clinicians in } \\
\text { IPV field }\end{array}$ \\
\hline & $\begin{array}{l}\text { •Conducted with } \\
\text { adolescents (adolescent } \\
\text { dating violence was } \\
\text { excluded) }\end{array}$ \\
\hline & $\begin{array}{l}\text { - Conducted with women } \\
\text { (studies which assessed } \\
\text { gender differences, } \\
\text { exclusively based on self- } \\
\text { report of battered women } \\
\text { or conducted with female } \\
\text { IPV perpetrators were } \\
\text { excluded) }\end{array}$ \\
\hline & $\begin{array}{l}\text { - Conducted with children } \\
\text { exposed to IPV or paternal } \\
\text { antisocial personality }\end{array}$ \\
\hline $\begin{array}{l}\text { Published (in any } \\
\text { language) before } \\
\text { September } 2017\end{array}$ & \\
\hline $\begin{array}{l}\text { Conducted in any setting } \\
\text { (e.g., inmates, intervention } \\
\text { programs participants or } \\
\text { community sample) }\end{array}$ & \\
\hline $\begin{array}{l}\text { Studies which assessed } \\
\text { psychopathy in adulthood }\end{array}$ & \\
\hline
\end{tabular}


batterers" OR "Male perpetrator" OR "Spouse abuse" OR "Family Violence" OR "Domestic Violence"). Search strategies were adapted to the different databases (see Figure 1 for the search outputs used in each database). An additional nine relevant articles were selected through a manual search of authors' personal collections. These were not previously identified because neither their author nor the databases had provided keywords related to psychopathy. Researches support handsearching efforts as supplemental approach to increase the quality of systematic reviews (Richards, 2008; Vassar, Atakpo, \& Kash, 2016). The literature search in this review comprised 41 articles from 1988 to 2017 . The last update was performed in December 2017. For an overview of the study selection process, see Figure 1.

\section{- CLASSIFICATION OF OUTCOMES}

The role of psychopathic traits in male-tofemale IPV perpetration was analyzed according to three criteria: (a) the aim of the studies, depending on whether the role of psychopathy to predict IPV perpetration or to establish batterer typologies was analyzed, (b) the type of samples used -non-convicted partner-violent men from community/clinical samples or convicted male batterers from forensic/prison samples-, and (c) the approach used to measure psychopathy -according to Hare's model of psychopathy or alternative psychopathy assessment tools-.

\section{RESULTS}

\section{-DESCRIPTION OF THE INCLUDED STUDIES}

AIM OF STUDIES. Twenty-six studies examined the role of psychopathy in predicting IPV perpetration, namely, either the differences between IPV and non-IPV men on psychopathic traits were investigated or the predictive power of psychopathy on IPV perpetration was analyzed. Additionally, nineteen studies analyzed the role of psychopathic traits to establish or differentiate among batterer typologies, namely, batterer subtypes were compared on psychopathic traits (see Table 2).

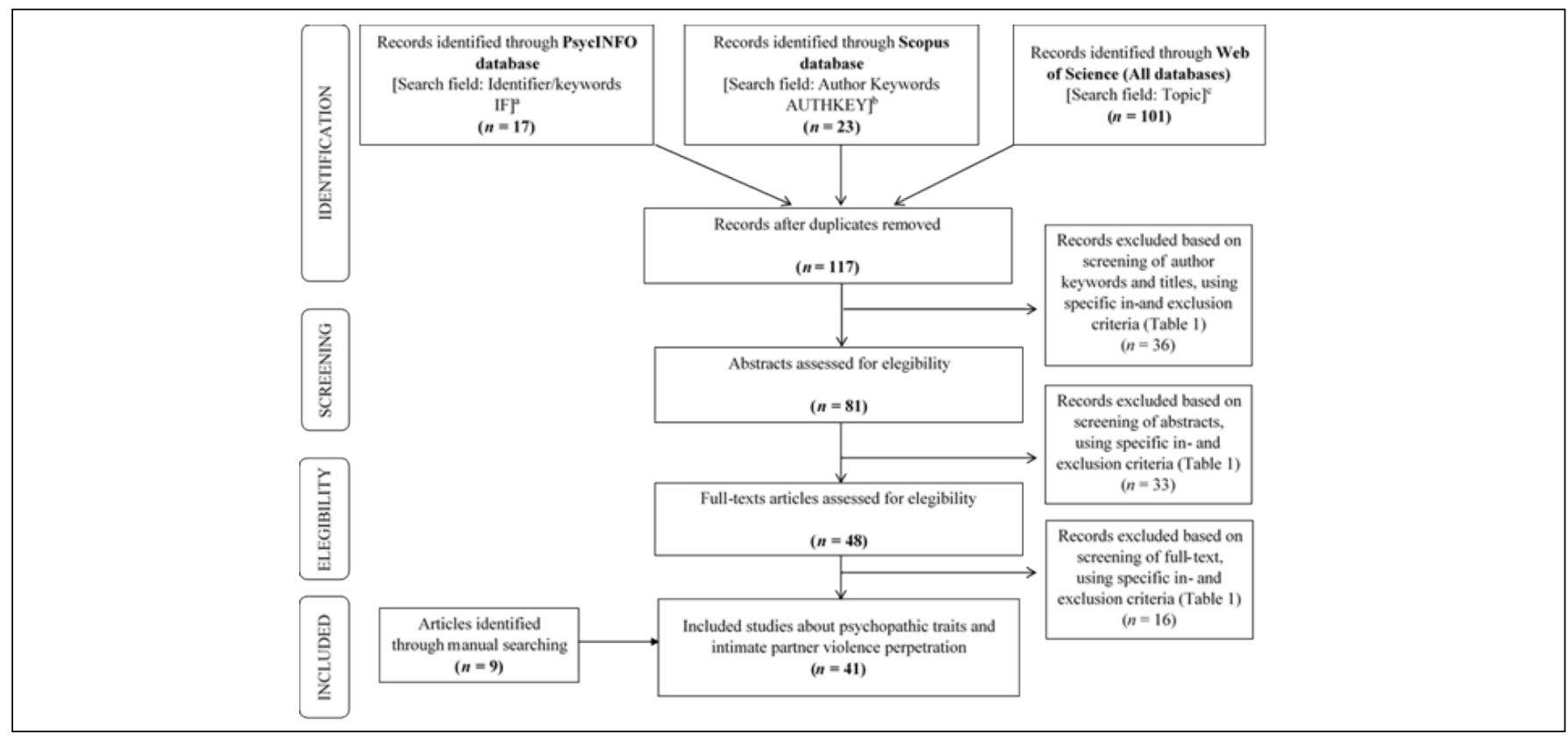

Figure 1: Summary of study selection process. "The search output used in PsycINFO: (if ("Psychopathy" OR "Psychopathic") AND if ("Intimate Partner Violence" OR "Partner abuse" OR "Male batterers" OR "Male perpetrators" OR "Spouse abuse" OR "Family violence" OR "Domestic violence" OR "IPV")) AND rtype.exact ("Journal Article"). 'The search output used in Scopus: AUTHKEY ("Psychopathy" OR "Psychopathic") AND AUTHKEY ("Intimate Partner Violence" OR "Partner abuse" OR "Male batterers" OR "Male perpetrators" OR "Spouse abuse" OR "Family violence" OR "Domestic violence" OR "IPV") AND ( LIMIT-TO (DOCTYPE, "ar" )). "The search output used in WoS (All databases): Topic: ("Psychopathy" OR "Psychopathic") AND Topic: ("Intimate Partner Violence"OR "Partner abuse" OR "Male batterers" OR "Male perpetrators" OR "Spouse abuse" OR "Family violence" OR "Domestic violence" OR "IPV"), Refined by: Type of document: (ARTICLE) 
Table 2

Description of included studies $(N=41)$

\begin{tabular}{|c|c|c|}
\hline & $n$ & $\%$ \\
\hline \multicolumn{3}{|l|}{ Aim of studiesa } \\
\hline To examine the role of psychopathy in predicting IPV perpetration & 26 & 63.41 \\
\hline To examine the role of psychopathy in establishing batterer typologies & 19 & 46.34 \\
\hline \multicolumn{3}{|l|}{ Sample of studies } \\
\hline Non-convicted partner-violent men from community or clinical samples & 20 & 48.78 \\
\hline $\begin{array}{l}\text { Convicted male batterers on probation or sentenced for IPV to prison or BIPs from forensic or prison } \\
\text { samples }\end{array}$ & 21 & 51.22 \\
\hline \multicolumn{3}{|l|}{ Approach of psychopathy measurement } \\
\hline $\begin{array}{l}\text { Psychopathy assessment tools according Hare's model (including antisocial traits as diagnostic } \\
\text { criteria) }\end{array}$ & 34 & 82.93 \\
\hline Alternative psychopathy assessment tools (excluding antisocial traits as diagnostic criteria) & 7 & 17.07 \\
\hline \multicolumn{3}{|l|}{ Language of studies } \\
\hline English & 40 & 97.56 \\
\hline Spanish & 1 & 2.44 \\
\hline
\end{tabular}

SAMPLES OF STUDIES. Twenty studies were conducted with non-convicted partner-violent men from community or clinical samples. In this case, the veracity of IPV depended on self- or partner-report measures. Another twenty-one studies were conducted with convicted male batterers on probation or sentenced for IPV to prison or BIPs from forensic or prison samples. Unlike in the previous group, in this case, IPV had been proven through judicial procedure (see Table 2).

Language of studies. Most studies (n $=40$ ) was written in English (see Table 2). The remaining study was written in Spanish (Fernández-Montalvo \& Echeburúa, 2008).

Approach of psychopathy measurement. Due to the observed variability with respect to the measurement of this construct, psychopathy assessment tools were analyzed more exhaustively (see Table 2). Most studies measured psychopathy using assessment tools that included antisocial traits as diagnostic criteria. Specifically, scales derived from the PCL-R were the most common psychopathy measurement. The PCL-R (Hare, 2003) was used by $10(24 \%)$ of the included studies. Nevertheless, only Swogger et al. (2007, 2012) scored PCL-R items from semistructured interviews and file review, as recommended by the PCL-R manual (Hare,
2003). Old and different versions of SRP (Neal \& Sellbom, 2012) were used in 9 studies (22\%), and the PCL-SV (Hart et al., 1995) was used by 5 (12\%) of the included studies. Again, only Huss and Langhinrichsen-Rohling (2006) and Mager et al. (2014) followed the PCL-SV manual's recommendations (Hart et al., 1995). Apart from PCL-R and its derivatives, other self-report assessment tools and traditional personality inventories were also used to assess psychopathy, including antisocial traits as diagnostic criteria.

In contrast, only seven studies measured psychopathy excluding antisocial traits as diagnostic criteria. The Psychopathic Personality Inventory (PPI; Lilienfeld \& Hess, 2001) and the Youth Psychopathic trait Inventory (YPI; Andershed, Kerr, Stattin, \& Levander, 2002) were used in three of the included studies. Other researchers decided to exclude items related to criminality/ antisociality from conventional psychopathy assessment tools (Babcock et al., 2008; Marshall and Holtzworth-Munroe, 2010; Walsh et al., 2010; Wymbs et al., 2017). It is remarkable that only one study conducted with a convicted sample (Stanford et al., 2008) excluded antisocial traits as diagnostic criteria. Studies that measured psychopathy excluding antisocial features also reported a positive significant association between 
psychopathy and IPV perpetration, which supported the role of core psychopathic traits (regardless of antisocial traits) on IPV perpetration.

Additionally, the majority of studies conceptualized the psychopathy in dimensional terms. Only ten studies (Belfrage \& Rying, 2004; Chase et al. , 2001 ; Echeburúa \& Fernández-Montalvo, 2007; FernándezMontalvo \& Echeburúa, 2008; Flournoy \& Wilson, 1991; Gondolf \& White, 2001 ; Hale et al., 1988; Huss \& Langhinrichsen-Rohling, 2006; Juodis et al., 2014a; Kalichman, 1988) used a categorical approach and provided data on the prevalence of psychopathic traits. Great variability was found regarding the prevalence rates of psychopathy, which ranged from 4 to $56 \%$, due to methodological differences between the studies, such as the variety of psychopathy assessment tools, the different cut-off scores assumed, and the unequal severity level of the samples (IPV homicide perpetrators vs. batterers in BIPs).

\section{- THE ROLE OF PSYCHOPATHY TO PREDICT MALE-TO-FEMALE IPV PERPETRATION}

Twenty-six studies examined the role of psychopathy to predict IPV perpetration among non-convicted partner-violent men (see Table 3; Babcock, Green, \& Webb, 2008; Babcock, Green, Webb, \& Yerington, 2005; Bates, Archer, \& Graham-Kevan, 2017; Coyne, Nelson, Graham-Kevan, Keister, \& Grant, 2010; Holtzworth-Munroe, Meehan, Herron, Rehman, \& Stuart, 2000; lyican, Sommer, Kini, \& Babcock, 2015; Kiire, 2017; Mager, Bresin, \& Verona, 2014; Marshall \& Holtzworth-Munroe, 2010; Okano, Langille, \& Walsh, 2016; Panuzio et al., 2006; Theobald, Farrington, Coid, \& Piquero, 2015; Thornton, Graham-Kevan, \& Archer, 2016; Walsh et al., 2010; Wymbs, Dawson, Suhr, Bunford, \& Gidycz, 2017) and convicted male batterers (see Table 4; Cunha \& Gonçalves, 2013; Harris, Hilton, \& Rice, 2011 ; Hilton, Harris, \& Rice, 2001; Hilton, Harris, Rice, Houghton, \& Eke, 2008; Hornsveld, Bezuijen, Leenaars, \& Kraaimaat, 2008; Juodis, Starzomski, Porter, \& Woodworth, 2014a; Kalichman, 1988; Rock, Sellbom, Ben-Porath, \& Salekin, 2013; Swogger, Walsh, \& Kosson, 2007; Swogger,
Walsh, Kosson, Cashman-Brown, \& Caine 2012; Taft, Murphy, Musser, \& Remington, 2004).

\section{- EVIDENCE FROM STUDIES CONDUCTED WITH NON-CONVICTED PARTNER-VIOLENT MEN}

Overall, studies underscored a significant positive small-to-moderate correlation (.19 $\leq r \leq$ .39) between psychopathy and IPV perpetration (Babcock et al., 2005; Bates et al., 2017; Coyne et al., 2010; lyican et al., 2015; Kiire, 2017; Marshall \& Holtzworth-Munroe, 2010; Thornton et al., 2016).

When more complex analyses were carried out, similar findings were found regarding the role of global psychopathy as a predictor of IPV perpetration (Kiire, 2017; Okano et al., 2016). Fast life history strategies (Kiire, 2017) mediated the relationship between psychopathy and IPV perpetration: psychopaths tend to establish shortterm relationships, which in turn were associated with involvement in IPV (Kiire, 2017). However, psychopathy did not impact the level of interpartner agreement for physical and psychological IPV (Panuzio et al., 2006).

Regarding the so-called core personality psychopathic traits, interpersonal and affective psychopathic traits seemed to predict IPV perpetration (Marshall \& Holtzworth-Munroe, 2010). Diminished sensitivity to their female partners' expressions of happiness mediated the relationship between core psychopathic traits and IPV perpetration (Marshall \& HoltzworthMunroe, 2010): Psychopaths labelled neutral and positive emotions as provocative, which were associated with IPV perpetration. Contradictory findings were found about the predictive capability of core psychopathic traits on specific physical IPV perpetration. Some studies suggested that high levels of interpersonal/ affective psychopathic traits were associated with physical IPV perpetration (Bates et al., 2017; Mager et al., 2014). However, when men's anger and self-control (Thornton et al., 2016) and exposure to media aggression (Coyne et al., 2010) were controlled, Factor 1 did not show an effect on physical IPV perpetration. Coyne et al. (2010) revealed an association between primary psychopathy and psychological but not physical IPV perpetration. In contrast, Wymbs et al. (2017) found the opposite result: primary 
psychopathy was associated with physical but not psychological IPV perpetration.

Regarding the behavioral psychopathic traits, all studies reported a relationship between these lifestyle/antisocial psychopathic traits and physical IPV perpetration (Bates et al., 2017; Coyne et al., 2010; Mager et al., 2014). Additionally, a strong association between high levels of Factor 2 of psychopathy and mutually physical IPV was underscored (Mager et al., 2014). Conflicting findings were found when specific lifestyle traits were analyzed separately (Theobald et al., 2015; Thornton et al., 2016).

Finally, when the levels of psychopathic traits were compared between partner-violent men and non-violent men, conflicting results were achieved: while Babcock et al. (2005, 2008), Holtzworth-Munroe et al. (2000), and Walsh et al. (2010) failed to demonstrate the utility of psychopathy to identify partner-violent men among community samples (non-violent men were as psychopathic as some subtype of partner-violent men), Theobald et al. (2015) reported higher levels of psychopathic traits among partner-violent than non-violent men.

\section{- EVIDENCE FROM STUDIES CONDUCTED WITH CONVICTED MALE BATTERERS}

Again, among convicted male batterers, most studies evidenced a significant positive small-to-moderate correlation $(.22 \leq r \leq .39)$ between psychopathy and male-to-female IPV perpetration (Harris et al., 2011 ; Hilton et al., 2008; Rock et al., 2013; Taft et al., 2004).

The majority of these studies aimed to compare male batterers with other offenders who had never committed offenses against their partners. Apparently contradictory results were identified (Hilton et al., 2001; Kalichman, 1988), probably due to methodological differences between the reviewed studies related to the variety of psychopathy assessment tools or the lack of prior equality between groups in terms of their level of generalized violence. When batterers were matched to other violent offenders, research suggested that IPV perpetrators displayed lower levels of psychopathy than the other offenders (Hilton et al., 2001; Juodis et al., 2014a). However, these differences could actually be due to differences in Factor 2 but not Factor 1 of psychopathy
(Hornsveld et al., 2008); that is, batterers could have exhibited the same core psychopathic traits but fewer behavioral psychopathic features than other violent offenders. This hypothesis would be consistent with Swogger et al. (2007), who, after controlling violent charges and matching the sample in terms of antisocial traits, found that high levels of affective traits and low levels of lifestyle psychopathic traits predicted IPV perpetration, namely, batterers displayed a more adaptive lifestyle than non-batterers. Later, Swogger et al. (2012) evidenced a significant relationship between childhood physical abuse victimization and adulthood IPV perpetration at moderate-high levels of lifestyle psychopathic traits.

\section{- THE ROLE OF PSYCHOPATHY IN BATTERER TYPOLOGIES}

Nineteen studies examined the role of psychopathy to establish batterer typologies or differentiate among batterer subtypes using samples composed of non-convicted partnerviolent men (see Table 3; Babcock, Green, Webb, \& Graham, 2004; Babcock et al., 2005, 2008; Chase, O'Leary, \& Heyman, 2001; Hale, Zimostrad, Duckworth, \& Nicholas, 1988; Holtzworth-Munroe, Meehan, Herron, Rehman, \& Stuart, 2003; Holtzworth-Munroe et al., 2000; Meehan, Holtzworth-Munroe, \& Herron, 2001; Theobald et al., 2015; Walsh et al., 2010) and convicted male batterers (see Table 4; Cunha \& Gonçalves, 2013; Echeburúa \& FernándezMontalvo, 2007; Eckhardt, Samper, \& Murphy, 2008; Fernández-Montalvo \& Echeburúa, 2008; Gondolf \& White, 2001; Huss \& LanghinrichsenRohling, 2006; Lawson, Brossart, \& Shefferman, 2010; Murphy, Taft, \& Eckhardt, 2007; Stanford, Houston, \& Baldridge, 2008).

\section{- EVIDENCE FROM STUDIES CONDUCTED WITH NON-CONVICTED PARTNER-VIOLENT MEN.}

Psychopathy was commonly used to examine the differences among batterer subtypes. Psychopathy failed to differentiate among batterer subtypes according the traditional batterer typologies. First, both Babcock et al. (2004) and Meehan et al. (2001) reported that psychopathy was not a useful variable to differentiate between the type 1 and type 2 batterers proposed by 
Table 3

Empirical quantitative studies about the role of psychopathic traits on intimate partner violence (IPV) perpetration among non-convicted parter-violent men ( $n=20 ; 49 \%$ of the included studies)

\begin{tabular}{|c|c|c|c|}
\hline Paper & Sample & Assessment & Relevant findings \\
\hline Babcock et al. (2004) & $\begin{array}{l}\text { Couples }(N=102) \text { from community } \\
\text { sample } \\
\text { - Target groups: clinical } \\
\text { ( } n=50 \text { ) and low ( } n=22 \text { ) level violent men } \\
\text { - Control group: non intimate partner- } \\
\text { violent men ( } n=30) \\
\text { Target and control groups were created } \\
\text { based on both self- and female partner- } \\
\text { reports on male-to-female IPV (CTS2): } \\
\text { clinical level (at least six minor, two } \\
\text { moderate or one severe male-to-female } \\
\text { IPV act in the past year), low level (at least } \\
\text { one minor o moderate male-to-female IPV } \\
\text { act in the past year), and control group } \\
\text { (no male-to-female IPV in the past } 5 \text { years } \\
\text { and no serious violence ever) } \\
100 \% \text { male (couples) } \\
\text { Mean men's age (SD): } 32 \text { (9.6) }\end{array}$ & $\begin{array}{l}\text { P: SRP-II } \\
\text { IPV: CTS2 (self- and } \\
\text { female partner- } \\
\text { reports) }\end{array}$ & $\begin{array}{l}\text { Clinical level violent men were } \\
\text { classified as heart reat decelerators } \\
\text { (type } 1 \text { batterers; } n=9 \text { ) and heart reat } \\
\text { accelerators (type } 2 \text { batterers; } n=41 \text { ) } \\
\text { based on heart reat reactivity during } \\
\text { marital interaction. } \\
\text { Role of psychopathic traits to differentiate } \\
\text { among batterer subtypes: } \\
\text { Not significant differences between type } \\
1 \text { and type } 2 \text { batterers on psychopathy. } \\
\text { MANOVA }\end{array}$ \\
\hline Babcock et al. (2005) & $\begin{array}{l}\text { Couples }(N=102) \text { from community } \\
\text { samplea } \\
\text { - Target group: severely ( } n=35 \text { ) and low- } \\
\text { level ( } n=37) \text { violent men } \\
\text { - Control group: non intimate partner- } \\
\text { violent men ( } n=30) \\
\text { Target and control groups were created } \\
\text { based on both self- and female partner- } \\
\text { reports on male-to-female IPV (CTS2): } \\
\text { severely violent (at least one severe } \\
\text { male-to-female IPV act in the past year), } \\
\text { low level (at least one no severe male- } \\
\text { to-female IPV act in the past year), and } \\
\text { control group (no male-to-female IPV in } \\
\text { the past } 5 \text { years and no serious violence } \\
\text { ever) } \\
100 \% \text { male (couples) } \\
\text { Mean men's age (SD): } 32 \text { (9.6) }\end{array}$ & $\begin{array}{l}\text { P: SRP-II } \\
\text { IPV: CTS2 (self- and } \\
\text { female partner- } \\
\text { reports) }\end{array}$ & $\begin{array}{l}\text { Predictive power of psychopathic traits } \\
\text { on IPV perpetration: } \\
\text { Significant correlation between } \\
\text { psychopathy and physical IPV partner- } \\
\text { reported ( } r=.23 ; p<.05) \text {, physical } \\
\text { IPV self-reported ( } r=.33 ; p<.01) \text {, and } \\
\text { psychological IPV self-reported }(r=.39 ; \\
p<.001) \text {, but not psychological IPV } \\
\text { partner-reported ( } r=.19 ; p=\text { n.s.). } \\
\text { Bivariate correlations } \\
\text { Not significant differences between non- } \\
\text { IPV men and the two IPV men subgroups } \\
\text { and on psychopathy, } F(2,97)=1.25 \text {, } p \\
=n \text {. s. } \\
\text { MANOVA, controlled for age and marital } \\
\text { satisfaction } \\
\text { Role of psychopathic traits to differentiate } \\
\text { among batterer subtypes: } \\
\text { Not significant differences between } \\
\text { severely violent and low-level violent } \\
\text { batterers on psychopathy, F( } 2,97)= \\
1.25, p=n \text {. s. } \\
\text { MANOVA, controlled for age and marital } \\
\text { satisfaction }\end{array}$ \\
\hline Babcock et al. (2008) & $\begin{array}{l}\text { Couples }(N=101) \text { from community } \\
\text { samplea } \\
\text { - Target group: domestically violent men } \\
\text { ( } n=69) \\
\text { - Control group: non intimate partner- } \\
\text { violent men ( } n=32) \\
\text { Target and control groups were created } \\
\text { based on both self- and female partner- } \\
\text { reports on male-to-female IPV (CTS2): } \\
\text { target group (one male-to-female IPV act } \\
\text { in the past year) and control group (no } \\
\text { male-to-female IPV in the past } 5 \text { years } \\
\text { and no serious violence ever) } \\
\text { 100\% male (couples) } \\
\text { Mean men's age (SD): } 32 \text { (9.6) }\end{array}$ & $\begin{array}{l}\text { P: SRP-II } \\
\text { IPV: CTS2 (self- and } \\
\text { female } \\
\text { reports) }\end{array}$ & $\begin{array}{l}\text { Predictive power of psychopathic traits } \\
\text { on IPV perpetration: } \\
\text { Not significant differences between non- } \\
\text { IPV men and the all IPV men subgroups } \\
\text { on psychopathy Factor } 1 \text {. } \\
\text { MANOVA } \\
\text { Role of psychopathic traits to establish } \\
\text { batterer typologies: } \\
\text { Target group were classified based } \\
\text { on CTS2 partner-report, borderline } \\
\text { personality, psychopathy Factor } 1 \text {, and } \\
\text { general violence: FO ( } n=43 \text { ), BD ( } n= \\
\text { 14) and GVP ( } n=12 \text { ) batterers. } \\
\text { Cluster analytic techniques } \\
\text { Significant differences between BD and } \\
\text { GVP batterers on psychopathy Factor } 1 \text {, } \\
F(3,97)=6.17, p<.01 \text { (GVP }>\text { BD). } \\
\text { MANOVA }\end{array}$ \\
\hline
\end{tabular}


Table 3 (Continuation)

Empirical quantitative studies about the role of psychopathic traits on intimate partner violence (IPV) perpetration among non-convicted parter-violent men ( $n=20 ; 49 \%$ of the included studies)

\begin{tabular}{|c|c|c|c|}
\hline Paper & Sample & Assessment & Relevant findings \\
\hline Bates et al. (2017) & $\begin{array}{l}\text { STUDY 3: } \\
\text { University sample }(N=364) \\
\text { Non control group composed by non IPV } \\
\text { perpetrators } \\
44 \% \text { male } \\
\text { Mean men's age }(S D): 25.78(n r)\end{array}$ & $\begin{array}{l}\text { P: LSRP } \\
\text { IPV: CTS physical } \\
\text { aggression scale }\end{array}$ & $\begin{array}{l}\text { RESULTS FOR MEN: } \\
\text { Predictive power of psychopathic traits } \\
\text { on IPV perpetration: } \\
\text { Significant correlation between } \\
\text { physical IPV perpetration and primary } \\
\text { psychopathy }(r=.27 ; p<.001 \text { ), and } \\
\text { secondary psychopathy }(r=.26 ; p< \\
.001 \text { ). } \\
\text { Zero-Order correlations } \\
\text { Significant main effect of primary (only } \\
\text { for men), and secondary (for both } \\
\text { men and women, } B=.14 \text {, SE }=.04 \text {, } \\
\chi^{2}=10.97, p<.05-\text { not significant } \\
\text { interaction between sex and secondary } \\
\text { psychopathy-) psychopathy on } \\
\text { physical IPV perpetration. } \\
\text { Negative binomial regression analysis }\end{array}$ \\
\hline Chase et al. (2001) & $\begin{array}{l}\text { Clinical sample composed by men who } \\
\text { were self-referred to BIP }(N=60) \\
\text { Not control group composed by non IPV } \\
\text { perpetrators } \\
100 \% \text { male } \\
\text { Mean age }(S D) \text { : nr }\end{array}$ & $\begin{array}{l}\text { P: dichotomous } \\
\text { score from MCMI- } \\
\text { II (narcissistic and } \\
\text { antisocial subscales) } \\
\text { IPV: CTS (all } \\
\text { participants had } \\
\text { reported } 2 \text { male-to- } \\
\text { female IPV acts in the } \\
\text { past year) }\end{array}$ & $\begin{array}{l}\text { Prevalence of psychopathy among } \\
\text { male batterers in BIP: } 17 \% \text { (based on } \\
\text { MCMI-II Narcissistic and Antisocial base } \\
\text { rate scores }>85 \text { ). } \\
\text { Partner-violent men were classified as } \\
\text { reactive ( } n=37 \text { ) and proactive ( } n= \\
23 \text { ) based on impulsivity-intentionality of } \\
\text { IPV and affectivity/physiological arousal } \\
\text { prior to and during IPV. } \\
\text { Role of psychopathic traits to differentiate } \\
\text { among batterer subtypes: } \\
\text { Significant differences between reactive } \\
\text { and proactive batterers on psychopathy, } \\
\chi^{2}(1,60)=6.89, p<.01: 0 \% \text { of reactive, } \\
\text { and } 17 \% \text { of proactive IPV men were } \\
\text { classified as psychopaths. } \\
\text { Group comparison ( } \chi^{2} \text { test) }\end{array}$ \\
\hline Coyne et al. (2010) & $\begin{array}{l}\text { University sample ( } N=337) \\
\text { Non control group composed by non IPV } \\
\text { perpetrators } \\
45 \% \text { male } \\
\text { Mean men's age }(S D): \mathrm{nr}\end{array}$ & $\begin{array}{l}\text { P: LSRP } \\
\text { IPV: CTS physical } \\
\text { aggression scale, RRA }\end{array}$ & $\begin{array}{l}\text { RESULTS FOR MEN: } \\
\text { Predictive power of psychopathic traits } \\
\text { on IPV perpetration: } \\
\text { Significant correlation between primary } \\
\text { psychopathy and physical ( } r=.14, p \\
<.10) \text {, and psychological }(r=.26, p< \\
.01) \text { IPV. } \\
\text { Significant correlation between } \\
\text { secondary psychopathy and physical ( } r \\
=.25, p<.01) \text {, and psychological }(r= \\
.30, p<.001) \text { IPV. } \\
\text { Bivariate correlations } \\
\text { Significant relationship between primary } \\
\text { psychopathy and psychological ( } r= \\
.14, p<.10) \text { but not physical ( } r=.00, \\
p=n . s .) \text { IPV. } \\
\text { Significant relationship between } \\
\text { secondary psychopathy and physical }(r \\
=.21 ; p<.01) \text { and psychological }(r= \\
.20 ; p<.05) \text { IPV. } \\
\text { Multivariate multiple regressions analysis, } \\
\text { controlled for exposure to media } \\
\text { (physical and psychological) aggression } \\
\text { Exposure to media (physical and } \\
\text { psychological) aggression did not } \\
\text { mediated the relationship between } \\
\text { psychopathy and IPV. } \\
\text { Mediational analysis }\end{array}$ \\
\hline
\end{tabular}


Table 3 (Continuation)

Empirical quantitative studies about the role of psychopathic traits on intimate partner violence (IPV) perpetration among non-convicted parter-violent men ( $n=20 ; 49 \%$ of the included studies)

\begin{tabular}{|c|c|c|c|}
\hline Paper & Sample & Assessment & Relevant findings \\
\hline Hale et al. (1988) & $\begin{array}{l}\text { Clinical sample composed by men who } \\
\text { were self-referred to BIP }(N=67) \\
\text { Not control group composed by non IPV } \\
\text { perpetrators } \\
100 \% \text { male } \\
\text { Mean age (SD): } 31(\mathrm{nr})\end{array}$ & $\begin{array}{l}\text { P: MMPI } \\
\text { Psychopathic } \\
\text { deviate scale } \\
\text { IPV: all participants } \\
\text { had reported at } \\
\text { least one male-to- } \\
\text { female physically } \\
\text { IPV act }\end{array}$ & $\begin{array}{l}\text { Prevalence of psychopathic deviate } \\
\text { among male batterers in BIP: } 56 \% \text { (based } \\
\text { on T score > } 70 \text { on MMPI psychopathic } \\
\text { deviate scale). } \\
\text { The mos common MMPI profiles were the } \\
\text { combination of psychopathic deviate with } \\
\text { depression (19\%), schizophrenia ( } 12 \% \text { ), } \\
\text { hypomania ( } 9 \% \text { ), and hysteria ( } 7 \% \text { ). } \\
\text { Role of psychopathic traits to establish } \\
\text { batterer typologies: } \\
\text { Batterers were classified based on MMPI } \\
\text { validity and clinical (Hypochondriasis, } \\
\text { Depression, Hysteria, Psychopathic } \\
\text { Deviant, Paranoia, Psychoasthenia, } \\
\text { Schizophrenia, and Hypomania) scales: } \\
\text { cluster } 1 \text { ( } n=7 \text { ), cluster } 2 \text { ( } n=10 \text { ), and } \\
\text { cluster } 3 \text { ( } n=50 \text { ). } \\
\text { Cluster analytic techniques } \\
\text { Cluster } 3 \text { batterers displayed a MMPI profile } \\
\text { based on combination of psychopathic } \\
\text { deviate and depression. }\end{array}$ \\
\hline $\begin{array}{l}\text { Holtzworth-Munroe et al. } \\
\qquad(2000)\end{array}$ & $\begin{array}{l}\text { Couples }(N=164) \text { from community } \\
\text { samplea } \\
\text { - Target group: violent husbands ( } n=102) \\
\text { - Control group: nonviolent husbands } \\
\text { ( } n=62) \\
\text { Target and control groups were created } \\
\text { based on both self- and female partner- } \\
\text { reports on male-to-female physical IPV } \\
\text { (CTS2 item 12): target group (one male-to- } \\
\text { female physical IPV act in the past year) } \\
\text { and control group (no male-to-female } \\
\text { physical IPV act in the past } 5 \text { years and no } \\
\text { severe violence ever) } \\
100 \% \text { male (couples) } \\
\text { Mean men's age (SD): } 35.62 \text { ( } 9.26 \text { ) }\end{array}$ & $\begin{array}{l}\text { P: SRP } \\
\text { IPV: CTS-2, SES, } \\
\text { PMWI (self- and } \\
\text { female partner- } \\
\text { reports) }\end{array}$ & 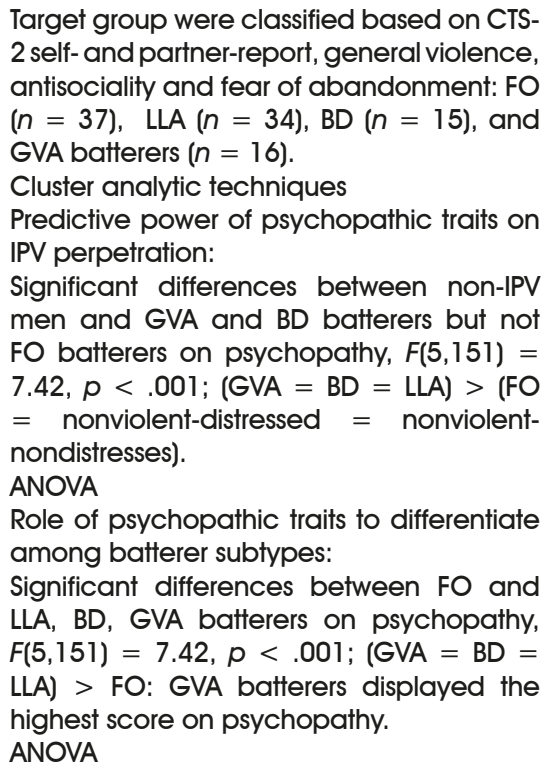 \\
\hline $\begin{array}{l}\text { Holtzworth-Munroe et al. } \\
\qquad(2003)\end{array}$ & $\begin{array}{l}\text { Violent couples ( } N=102 \text { at } \mathrm{T} 1 \text { ) from } \\
\text { Holtzworth-Munroe et al. }(2000) \\
\text { community samplea } \\
\text { Not control group composed by non IPV } \\
\text { perpetrators } \\
100 \% \text { male (couples) } \\
\text { Mean men's age (SD): } 36.02(9.52) \text { at } \mathrm{T} 1 \text {; } \\
\text { T2 and T3 were approximately } 1.5 \text { and } 3 \\
\text { years after } \mathrm{T} 1 \text {, respectively. }\end{array}$ & $\begin{array}{l}\mathrm{P} \text { (at T1 and T2): SRP } \\
\text { IPV (at T1, T2, and } \\
\text { T3): CTS-2, SES, PMWI } \\
\text { (self- and female } \\
\text { partner-reports) }\end{array}$ & $\begin{array}{l}\text { Four batterer subgroups were obtained at } \\
\text { T1 as Holtzworth-Munroe et al. ( } 2000) \text { : FO ( } n \\
=37), \text { LLA }(n=34), \mathrm{BD}(n=15), \text { and GVA } \\
\text { batterers }(n=16) \text {. } \\
\text { Cluster analytic techniques } \\
\text { Role of psychopathic traits to differentiate } \\
\text { among batterer subtypes: } \\
\text { Significant main effect of time on } \\
\text { psychopathy, } F(1,88)=5.00, p<.03 \text {; } \\
\text { across batterer clusters, psychopathy } \\
\text { decreased from T1 to T2. } \\
\text { Not significant interaction effect of Batterer } \\
\text { clusters x Time on psychopathy, } F(3,88)= \\
0.42, p=\text { n.s.; across T1 and T2, (GVA = BD } \\
=\text { LLA) > FO, GVA batterers displayed the } \\
\text { highest score on psychopathy. } \\
\text { ANOVA }\end{array}$ \\
\hline
\end{tabular}


Table 3 (Continuation)

Empirical quantitative studies about the role of psychopathic traits on intimate partner violence (IPV) perpetration among non-convicted parter-violent men $(n=20 ; 49 \%$ of the included studies)

\begin{tabular}{|c|c|c|c|}
\hline Paper & Sample & Assessment & Relevant findings \\
\hline Iyican et al. (2015) & $\begin{array}{l}\text { Couples }(N=114) \text { from } \\
\text { community samplea } \\
\text { - Target group: violent couples ( } n \\
=95) \\
\text { - Control group: non-violent } \\
\text { couples }(n=19) \\
\text { Target and control groups were } \\
\text { created based on female } \\
\text { partner`s reports on male-to- } \\
\text { female IPV (CTS-2): target group } \\
\text { (at least two male-to-female IPV } \\
\text { act in the past year) and control } \\
\text { group (distressed but non-violent) } \\
\text { 100\% male (couples) } \\
\text { Mean men's age (SD): } \mathrm{nr}\end{array}$ & $\begin{array}{l}\text { P: PPI-SF } \\
\text { IPV: CTS-2 } \\
\text { negotiation, } \\
\text { physical, and } \\
\text { psychological, } \\
\text { aggression scales } \\
\text { (self- and female } \\
\text { partner-reports) }\end{array}$ & 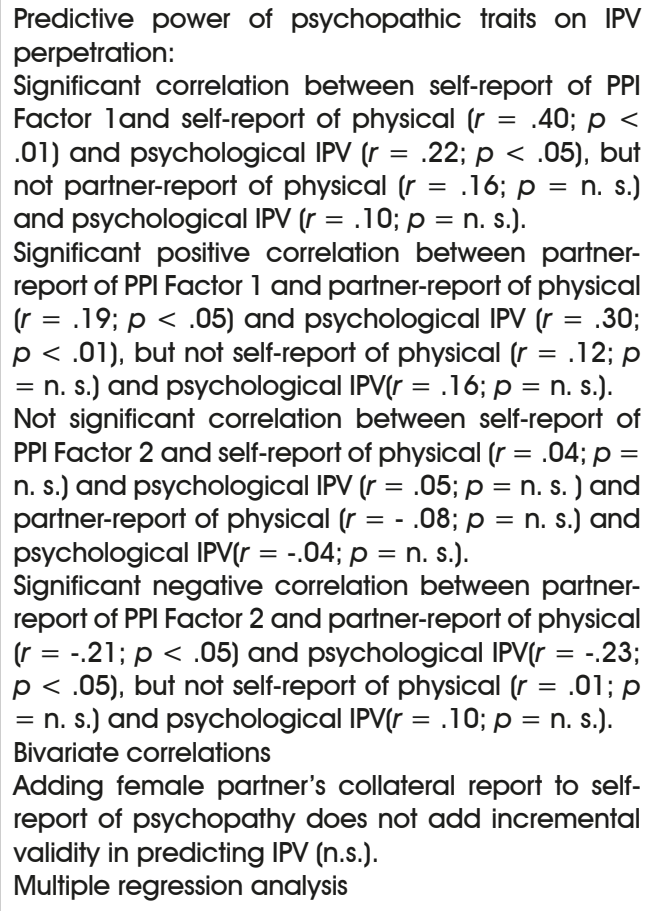 \\
\hline Kiire (2017) & $\begin{array}{l}\text { University sample }(N=344) \\
\text { Not control group composed by } \\
\text { non IPV perpetrators } \\
47.09 \% \text { male } \\
\text { Mean men's age }(S D): \mathrm{nr}\end{array}$ & $\begin{array}{l}\text { P: SD3J } \\
\text { psychopathy } \\
\text { subscale } \\
\text { IPV: IPV scale } \\
\text { perpetration } \\
\text { subscale }\end{array}$ & 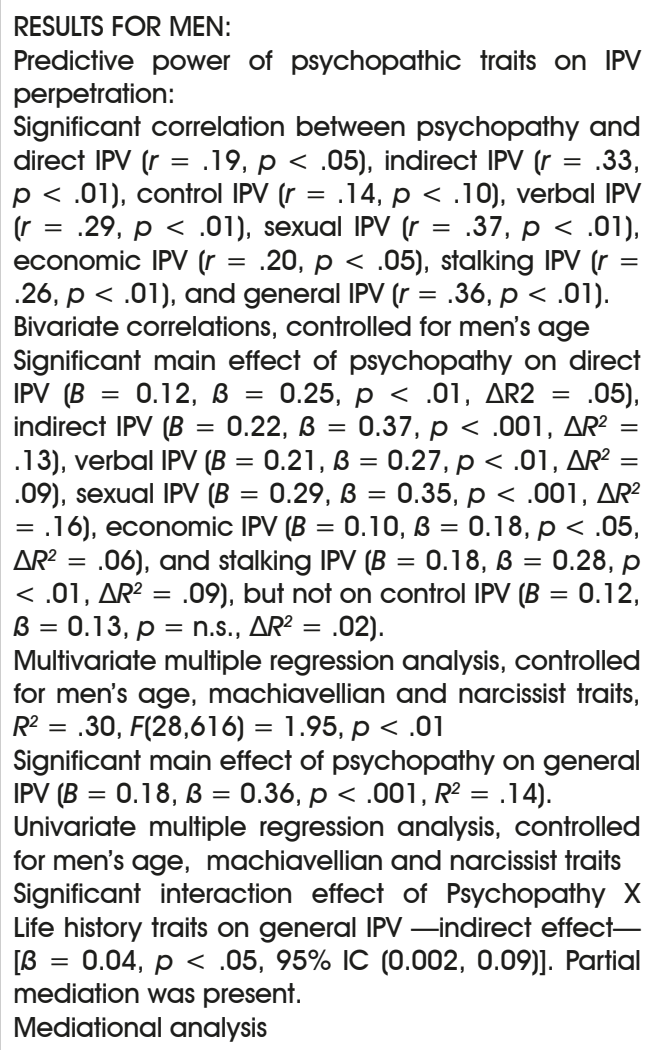 \\
\hline
\end{tabular}


Table 3 (Continuation)

Empirical quantitative studies about the role of psychopathic traits on intimate partner violence (IPV) perpetration among non-convicted parter-violent men ( $n=20 ; 49 \%$ of the included studies)

\begin{tabular}{|c|c|c|c|}
\hline Paper & Sample & Assessment & Relevant findings \\
\hline Mager et al. (2014) & $\begin{array}{l}\text { Clinical and community } \\
\text { sample recruited } \\
\text { from substance use } \\
\text { treatment or community } \\
\text { advertisement }(N=250) \\
\text { Not control group } \\
\text { composed by non IPV } \\
\text { perpetrators } \\
57 \% \text { male } \\
\text { Mean men's age }(S D): \mathrm{nr}\end{array}$ & $\begin{array}{l}\text { P: PCL-SV (from } \\
\text { interview + file } \\
\text { records) } \\
\text { IPV: CTS2 physical } \\
\text { aggression scale } \\
\text { (perpetration and } \\
\text { victimization) }\end{array}$ & 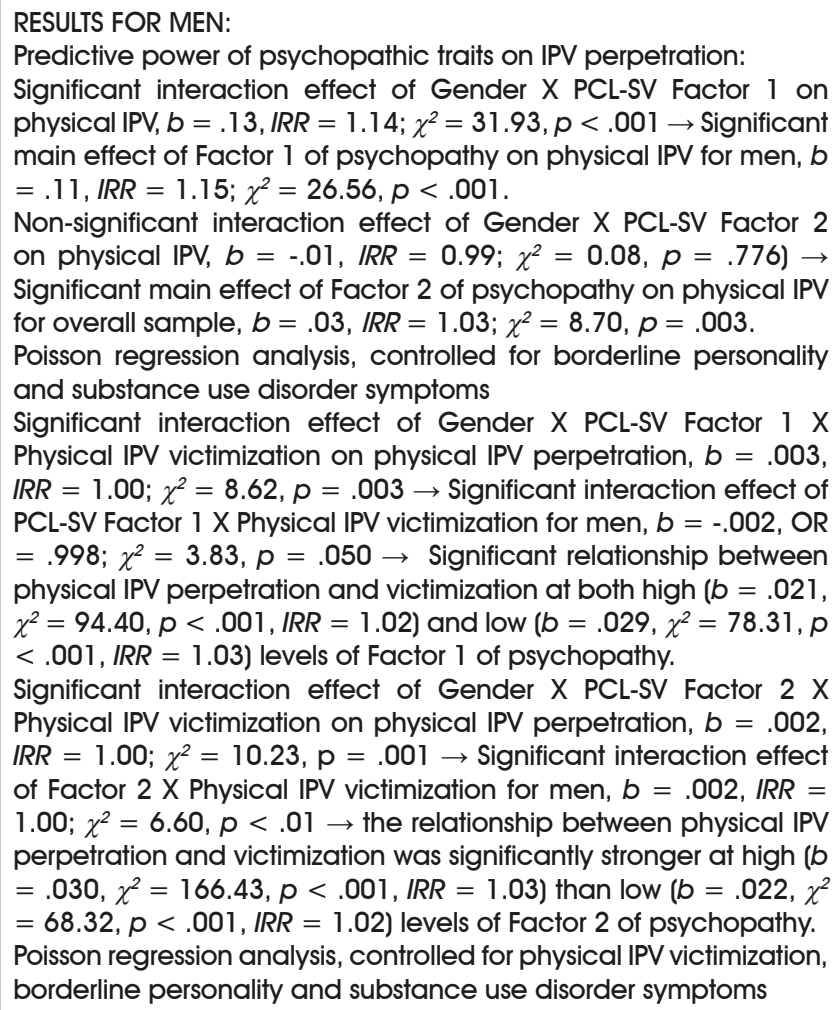 \\
\hline $\begin{array}{l}\text { Marshall and Holtzworth- } \\
\text { Munroe (2010) }\end{array}$ & $\begin{array}{l}\text { Couples }(N=88) \text { from } \\
\text { community sample } \\
\text { Not control group } \\
\text { composed by non IPV } \\
\text { perpetrators } \\
100 \% \text { male (couples) } \\
\text { Mean men's age (SD): } \\
37.1(9.4)\end{array}$ & $\begin{array}{l}\text { P: SRP, excluding } \\
\text { items related } \\
\text { to criminal } \\
\text { tendencies } \\
\text { IPV: CTS2 (self- } \\
\text { and female } \\
\text { partner-reports) }\end{array}$ & $\begin{array}{l}\text { Predictive power of psychopathic traits on IPV perpetration: } \\
\text { Significant correlation between psychopathy and IPV }(r=.28, p \\
=.01) \text {. } \\
\text { Bivariate correlations } \\
\text { Significant main effect of psychopathy on IPV perpetration, } \beta= \\
.28, p<.01 \text { - direct effect-. } \\
\text { Significant main effect of psychopathy on IPV perpetration through } \\
\text { sensitivity to wife's expression of fear -indirect effect-, } \beta=.24, p \\
<.05 \text {; partial mediation was not present. } \\
\text { Significant main effect of psychopathy on IPV perpetration through } \\
\text { sensitivity to unfamiliar women's expression of fear -indirect } \\
\text { effect-, } \beta=.24, p<.05 \text {; partial mediation was not present. } \\
\text { Not significant main effect of psychopathy on IPV perpetration } \\
\text { through sensitivity to wife's expression of happiness -indirect } \\
\text { effect }-, \beta=.19, p=\mathrm{n} . \mathrm{s} \text {; partial mediation was present. } \\
\text { Mediational analysis }\end{array}$ \\
\hline Meehan et al. (2001) & $\begin{array}{l}\text { Violent couples }(\mathrm{N}=58 \text { ) } \\
\text { from Holtzworth-Munroe } \\
\text { et al. (2000) who had } \\
\text { experienced moderate- } \\
\text { to-severe levels of IPV } \\
\text { based on CTS-2 (at least } \\
\text { six minor, two moderate } \\
\text { or one severe male-to- } \\
\text { female IPV act in the } \\
\text { past year) - community } \\
\text { samplea } \\
\text { Non control group } \\
\text { composed by non IPV } \\
\text { perpetrators } \\
\text { 100\% male (couples) } \\
\text { Mean men's age: nr }\end{array}$ & $\begin{array}{l}\text { P: SRP } \\
\text { IPV: CTS-2 (self- } \\
\text { and female } \\
\text { partner-reports) }\end{array}$ & $\begin{array}{l}\text { Violent men were classified as heart reat decelerators (type 1) } \\
\text { and heart reat accelerators (type } 2) \text { based on heart reat reactivity } \\
\text { during the first } 2.5 \text { min [type } 1(n=19) \text {; type } 2(n=39) \text { ] and } 5 \text { [type } \\
1(n=20) \text {; type } 2(n=38)] \text { min of marital interaction. } \\
\text { Role of psychopathic traits to differentiate among batterer } \\
\text { subtypes: } \\
\text { Not significant differences between type } 1 \text { and type } 2 \text { batterers } \\
\text { on psychopathy for } 2.5 \text { min reactivity }(p=n \text {. s.) and for } 5 \text { min } \\
\text { reactivity }(p=n \text {. s.). } \\
\text { Group comparisons (t test) }\end{array}$ \\
\hline
\end{tabular}


Table 3 (Continuation)

Empirical quantitative studies about the role of psychopathic traits on intimate partner violence (IPV) perpetration among non-convicted parter-violent men ( $n=20 ; 49 \%$ of the included studies)

\begin{tabular}{|c|c|c|c|}
\hline Paper & Sample & Assessment & Relevant findings \\
\hline \multirow[t]{2}{*}{ Okano et al. (2016) } & $\begin{array}{l}\text { STUDY 1: } \\
\text { Clinical sample }(N=703) \text { composed } \\
\text { by civil psychiatric patients } \\
\text { Non control group composed by non } \\
\text { IPV perpetrators } \\
58.75 \% \text { male } \\
\text { Mean men's age }(S D): \mathrm{nr}\end{array}$ & $\begin{array}{l}\text { P: PCL-SV } \\
\text { IPV: } \\
\text { combination } \\
\text { of self-report, } \\
\text { reports of } \\
\text { collateral } \\
\text { informants } \\
\text { and review of } \\
\text { official police } \\
\text { and hospital } \\
\text { records }\end{array}$ & $\begin{array}{l}\text { RESULTS WERE EQUIVALENT FOR MALES AND FEMALES: } \\
\text { Predictive power of psychopathic traits on IPV perpetration: } \\
\text { Significant main effect of psychopathy on IPV perpetration, } \\
\text { Exp }(B)=1.08 \text {, Wald }=22.14, p<.05, \Delta R^{2}=.04 \rightarrow 20.83 \% \\
\text { of the lower psychopathy group, and } 33.53 \% \text { of the higher } \\
\text { psychopathy group perpetrated IPV. } \\
\text { Not significant interaction effect of Psychopathy X Gender } \\
\text { on IPV perpetration, Exp }(B)=0.98 \text {, Wald }=0.47, p=\text { n.s., } \\
\Delta R^{2}<.01 \text {. } \\
\text { Multivariate Logistic Regression Analysis, controlled for } \\
\text { gender and alcohol use }\end{array}$ \\
\hline & $\begin{array}{l}\text { STUDY } 2 \text { : } \\
\text { University sample }(N=870) \\
\text { Non control group composed by non } \\
\text { IPV perpetrators } \\
17.24 \% \text { male } \\
\text { Mean men's age }(S D): \mathrm{nr}\end{array}$ & $\begin{array}{l}\text { P: SRP-4 } \\
\text { IPV: CTS2 } \\
\text { physical } \\
\text { aggression } \\
\text { scale }\end{array}$ & $\begin{array}{l}\text { RESULTS WERE EQUIVALENT FOR MALES AND FEMALES: } \\
\text { Predictive power of psychopathic traits on IPV perpetration: } \\
\text { Significant main effect of psychopathy on physical IPV } \\
\text { perpetration, Exp }(B)=3.14 \text {, Wald }=39.41, p<.05, \Delta R^{2}= \\
.06 \rightarrow 29 \% \text { of the lower psychopathy group, and } 42.60 \% \\
\text { of the higher psychopathy group perpetrated physical IPV. } \\
\text { Not significant interaction effect of Psychopathy X Gender } \\
\text { on physical IPV perpetration, Exp( }(B)=0.47 \text {, Wald }=1.80, p \\
=\text { n.s., } \Delta R^{2}<.01 \text {. } \\
\text { Multivariate Logistic Regression Analyses, controlled for } \\
\text { gender and alcohol use } \\
\text { Alcohol use scores did not mediate the association between } \\
\text { physical IPV perpetration and psychopathy }\left(z^{\prime}=1.91, p=\right. \\
\text { O6). } \\
\text { Mediational analysis }\end{array}$ \\
\hline Panuzio et al. (2006) & $\begin{array}{l}\text { Clinical sample }(N=303) \text { composed } \\
\text { by men recruited from alcoholism } \\
\text { treatment, and their female partnersa } \\
\text { Not control group composed by non } \\
\text { IPV perpetrators } \\
100 \% \text { male (couples) } \\
\text { Mean men's age }(S D): 43.3(10.0)\end{array}$ & $\begin{array}{l}\text { P: CPI } \\
\text { socialization } \\
\text { scale (higher } \\
\text { score, fewer } \\
\text { psychopathic } \\
\text { traits) } \\
\text { IPV: CTS } \\
\text { physical and } \\
\text { psychological } \\
\text { aggression } \\
\text { scales (self- } \\
\text { and female } \\
\text { partner- } \\
\text { reports) }\end{array}$ & $\begin{array}{l}\text { RESULTS FOR MEN: } \\
\text { Predictive power of psychopathic traits on IPV perpetration: } \\
\text { Significant negative correlation between CPI socialization } \\
\text { and inter-partner agreement for physical }(r=-.17 ; p<.05) \\
\text { and psychological IPV }(r=-.18 ; p<.01) \text {. } \\
\text { Zero-order correlations } \\
\text { Not significant partial association between psychopathy } \\
\text { and inter-partner agreement for physical }(r=-.01 ; p=n \text {. s.) } \\
\text { and psychological IPV }(r=.01 ; p=n \text {. } s \text {.). } \\
\text { Partial correlations, controlled for alcohol problems, } \\
\text { relationship adjustment, and antisocial personality disorder } \\
\text { Not significant main effect of psychopathy on inter-partner } \\
\text { agreement for physical }(\beta=-.02) \text { and psychological IPV ( } \beta \\
=.01) \text {. } \\
\text { Multiple regression analyses, controlled for alcohol problems, } \\
\text { relationship adjustment, and antisocial personality disorder }\end{array}$ \\
\hline $\begin{array}{l}\text { Theobald et al. } \\
\text { (2015) }\end{array}$ & $\begin{array}{l}\text { Couples ( } N=319 \text { ) from community } \\
\text { samplea } \\
\text { - Target group: IPV men ( } n=72 \text { ): } \rightarrow \\
\text { generally violent (both inside and } \\
\text { outside the home; } n=21 \text { ) and FO men } \\
(n=46 \text { ) } \\
\text { - Control group: non IPV men ( } n=247 \text { ) } \\
\rightarrow \text { violent convicted only }(n=23 \text { ) and } \\
\text { nonviolence ( } n=172 \text { ) group } \\
\text { Target and control group were } \\
\text { created based on combination of self- } \\
\text { (at } 32 \text { years) and partner- (at } 48 \text { years) } \\
\text { reports of physical IPV and information } \\
\text { on violent convictions } \\
100 \% \text { male (couples) } \\
\text { Mean age (SD): longitudinal study } \\
\text { (from } 8 \text { to } 48 \text { years). IPV was assessed } \\
\text { at } 32 / 48 \text { years. }\end{array}$ & $\begin{array}{l}\text { P: PCL-SV (from } \\
\text { file records } \\
\text { alone) } \\
\text { IPV: self-report } \\
\text { of physical } \\
\text { IPV, and CTS } \\
\mathrm{p} \text { h y s i c a I } \\
\text { aggression } \\
\text { scale (female } \\
\text { p a r t n e r's } \\
\text { reports) }\end{array}$ & 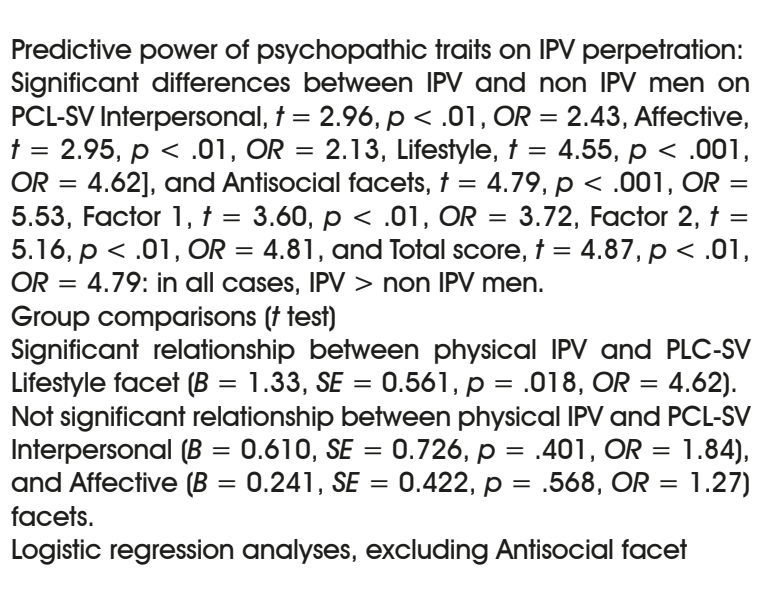 \\
\hline
\end{tabular}


Table 3 (Continuation)

Empirical quantitative studies about the role of psychopathic traits on intimate partner violence (IPV) perpetration among non-convicted parter-violent men ( $n=20 ; 49 \%$ of the included studies)

\begin{tabular}{|c|c|c|c|}
\hline Paper & Sample & Assessment & Relevant findings \\
\hline $\begin{array}{l}\text { Theobald } \\
\text { et al. (2015) } \\
\text { (Continuación) }\end{array}$ & $\begin{array}{l}\text { Couples }(N=319) \text { from community } \\
\text { samplea } \\
\text { - Target group: IPV men }(n=72): \rightarrow \\
\text { generally violent (both inside and } \\
\text { outside the home; } n=21 \text { ) and FO men } \\
(n=46) \\
\text { - Control group: non IPV men }(n=247) \\
\rightarrow \text { violent convicted only }(n=23) \text { and } \\
\text { nonviolence ( } n=172 \text { ) group } \\
\text { Target and control group were } \\
\text { created based on combination of self- } \\
\text { (at } 32 \text { years) and partner- (at } 48 \text { years) } \\
\text { reports of physical IPV and information } \\
\text { on violent convictions } \\
100 \% \text { male (couples) } \\
\text { Mean age (SD): longitudinal study } \\
\text { (from } 8 \text { to } 48 \text { years). IPV was assessed } \\
\text { at } 32 / 48 \text { years. }\end{array}$ & $\begin{array}{l}\text { P: PCL-SV (from } \\
\text { file records } \\
\text { alone) } \\
\text { IPV: self-report } \\
\text { of physical } \\
\text { IPV, and CTS } \\
\text { p h y s i a I } \\
\text { aggression } \\
\text { scale (female } \\
\text { partn e r's } \\
\text { reports) }\end{array}$ & 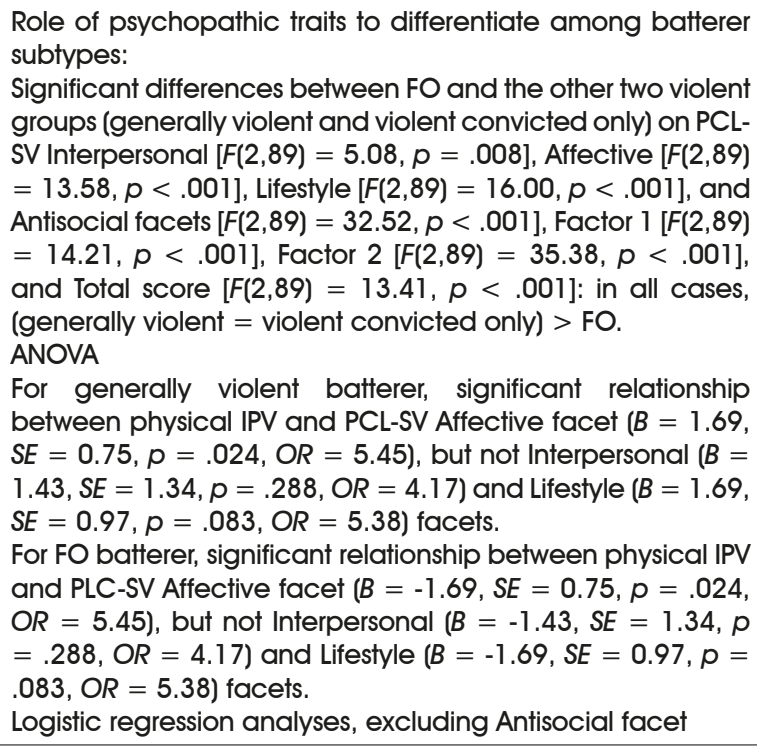 \\
\hline $\begin{array}{c}\text { Thornton et al. } \\
\text { (2016) }\end{array}$ & $\begin{array}{l}\text { University sample }(N=355) \\
\text { Not control group composed by non } \\
\text { IPV perpetrators } \\
51.83 \% \text { male } \\
\text { Mean men's age (SD): } 21.68(\mathrm{nr})\end{array}$ & $\begin{array}{l}\text { P: YPI } \\
\text { IPV: NVOBS } \\
\text { (physical IPV) }\end{array}$ & $\begin{array}{l}\text { RRESULTS FOR MEN: } \\
\text { Predictive power of psychopathic traits on IPV perpetration: } \\
\text { Significant correlation between physical IPV and Factor } \\
1(r=.35, p<.001) \text {, and Factor } 2(r=.26, p<.001) \text { of } \\
\text { psychopathy. } \\
\text { Bivariate correlations } \\
\text { Not significant main effect of Factor } 1(B=.01, S E=.01 \text {, } \\
\text { Wald } \chi^{2}=0.65, p=n . s \text {.) and Factor } 2(B=.04, S E=.03 \text {, } \\
\text { Wald } \chi^{2}=2.17, p=n . s \text {.) of psychopathy on physical IPV. } \\
\text { Not significant interaction effect of Factor } 1 \text { of Psychopathy } \\
X \text { Gender }\left(B=.03, S E=.01 \text {, Wald } \chi^{2}=3.54, p=n . s \text {.) and }\right. \\
\text { Factor } 2 \text { of Psychopathy X Gender ( } B=-.04, S E=.04 \text {, Wald } \\
\chi^{2}=0.92, p=n \text {.S.) on physical IPV. } \\
\text { Binomial Regression Analysis, controlled for gender, anger } \\
\text { and self-control }\end{array}$ \\
\hline Walsh et al. (2010) & $\begin{array}{l}\text { Clinical sample }(N=567) \text { composed } \\
\text { by civil psychiatric patients } \\
\text { - Target group: patients with history of } \\
\text { IPV perpetration }(n=231) \\
\text { - Control group: patients without } \\
\text { history of IPV perpetration }(n=336) \\
56.1 \% \text { male } \\
\text { Mean men's age }(S D): \mathrm{nr}\end{array}$ & $\begin{array}{l}\text { P: PCL-SV } \\
\text { (from interview } \\
\text { + file records) } \\
\text { IPV: } \\
\text { combination } \\
\text { of self-report, } \\
\text { reports of } \\
\text { collateral } \\
\text { informants, } \\
\text { and review of } \\
\text { official police } \\
\text { and hospital } \\
\text { records }\end{array}$ & $\begin{array}{l}\text { RESULTS FOR MEN: } \\
\text { Predictive power of psychopathic traits on IPV perpetration: } \\
\text { Not significant differences between non-IPV men and all } \\
\text { batterers subgroups on PCL-SV Total [Antisocial }>(\mathrm{LP}=\mathrm{BD} \\
=\text { control group)], Interpersonal [Antisocial }>\mathrm{LP}>(\mathrm{BD}= \\
\text { control group)], Affective facets [Antisocial }>(\mathrm{LP}=\mathrm{BD}= \\
\text { control group)], and Lifestyle facet scores [Antisocial }>\mathrm{BD}> \\
\text { (LP = control group)]. } \\
\text { MANOVA } \\
\text { Role of psychopathic traits to establish batterer typologies: } \\
\text { Target group were classifies based on PCL-SV Interpersonal, } \\
\text { Affective, and Lifestyle facets, traits of neuroticism, } \\
\text { extraversion, openness to experience, agreeableness and } \\
\text { conscientiousness: } \mathrm{LP}(n=51), \mathrm{BD}(n=25) \text {, and antisocials } \\
\text { ( } n=17) \text {. } \\
\text { Cluster analytic techniques among target group } \\
\text { Significant differences between antisocial and the other } \\
\text { subgroups (LP, BD and control group) on PCL-SV Total }[F \\
(3,314)=22.02, p=.01], \text { Interpersonal }[F(3,314)=23.95 \text {, } \\
p=.01] \text {, Affective facets }[F(3,314)=20.48, P=.01] \text {, and } \\
\text { Lifestyle facet scores }[F(3,314)=6.63, p=.01] \text { : Antisocial } \\
\text { batterers displayed significantly higher scores on PCL-SV } \\
\text { Total and all facets than other batterers subgroups. } \\
\text { MANOVA }\end{array}$ \\
\hline
\end{tabular}


Table 3 (Continuation)

Empirical quantitative studies about the role of psychopathic traits on intimate partner violence (IPV) perpetration among non-convicted parter-violent men $(n=20 ; 49 \%$ of the included studies)

\begin{tabular}{|c|c|c|c|}
\hline Paper & Sample & Assessment & Relevant findings \\
\hline Wymbs et al. (2017) & $\begin{array}{l}\text { University sample }(N=433) \\
37.2 \% \text { male } \\
\text { Mean men's age }(S D): \mathrm{nr}\end{array}$ & $\begin{array}{l}\text { P: LSRP } \\
\text { IPV: CTS-2 } \\
\text { physical and } \\
\text { psychological } \\
\text { aggression } \\
\text { scales }\end{array}$ & $\begin{array}{l}\text { RESULTS FOR MEN: } \\
\text { Predictive power of psychopathic traits on IPV perpetration: } \\
\text { Significant main effect of primary psychopathy on physical } \\
(\beta=.12, S E=0.4, p<.01) \text {, but not psychological IPV ( } \beta= \\
.08, S E=0.5, p=\text { n. } \mathrm{S} \text {.). } \\
\text { Regression analyses, controlled for gender, childhood } \\
\text { maltreatment, alcohol and drug abuse, and ADHD }\end{array}$ \\
\hline \multicolumn{4}{|c|}{ 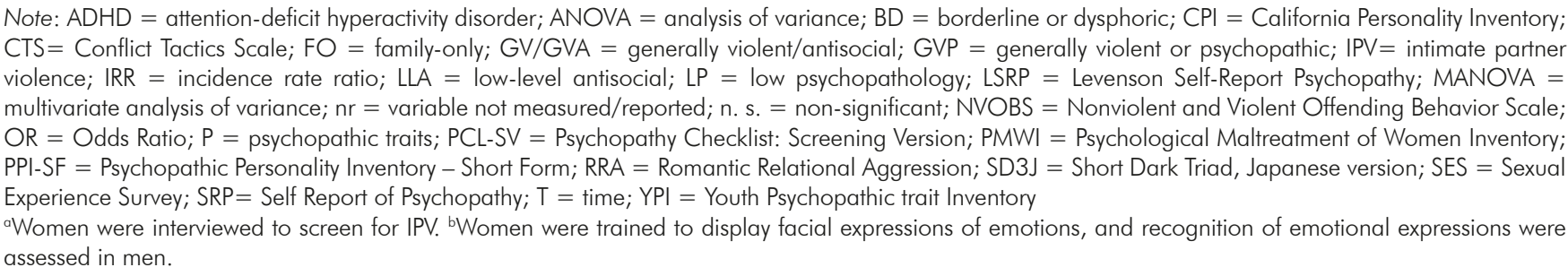 } \\
\hline
\end{tabular}

Table 4

Empirical quantitative studies about the role of psychopathic traits on intimate partner violence perpetration among convicted male batterers $(n=21$; $51 \%$ of the included studies)

\begin{tabular}{|c|c|c|c|}
\hline Paper & Sample & Assessment & Relevant findings \\
\hline $\begin{array}{l}\text { Belfrage and } \\
\text { Rying (2004) }\end{array}$ & $\begin{array}{l}\text { Forensic sample } \\
\text { - Target group: spousal homicide } \\
\text { perpetrators }(n=164) \\
\text { - Control group: homicide perpetrators } \\
\text { committed outside the context of } \\
\text { intimate partner relationship }(n=690) \\
\text { Target and control groups were } \\
\text { created based on material in police } \\
\text { investigations } \\
100 \% \text { male } \\
\text { Mean age }(S D): \mathrm{nr}\end{array}$ & $\begin{array}{l}\text { P: PCL-SV (from } \\
\text { file records } \\
\text { alone) } \\
\text { IPV: self-report } \\
\text { of physical } \\
\text { IPV, and CTS } \\
\mathrm{p} \text { h y s i c a I } \\
\text { aggression } \\
\text { scale (female } \\
\text { part n e r's } \\
\text { reports) }\end{array}$ & $\begin{array}{l}\text { Prevalence of psychopathy among intimate partner homicides: } \\
4 \% \text { (based on a cut-off of } 18 \text { on PCL-SV for diagnostic of } \\
\text { psychopathy). }\end{array}$ \\
\hline $\begin{array}{l}\text { Cunha and } \\
\text { Gonçalves } \\
\text { (2013) }\end{array}$ & $\begin{array}{l}\text { Prison sample composed by men } \\
\text { sentenced for IPV }(n=111) \text { and forensic } \\
\text { sample composed by convicted male } \\
\text { batterers in community }(n=76) \\
\text { Not control group composed by non } \\
\text { IPV perpetrators } \\
100 \% \text { male } \\
\text { Mean age (SD): } 43.81(11.23)\end{array}$ & $\begin{array}{l}\text { P: PCL-R (from } \\
\text { semi-structured } \\
\text { interview } \\
\text { alone) } \\
\text { IPV: IVC }\end{array}$ & $\begin{array}{l}\text { Role of psychopathic traits to establish batterer typologies: } \\
\text { Batterers were classified based on psychopatology (depression, } \\
\text { paranoid ideation, and PCL-R Affective and Antisocial facets), } \\
\text { physical and psychological IPV, hostility and generalized } \\
\text { physical violence: antisocial/violent }(n=51) \text {, non pathological } \\
(n=74) \text { and disturbed }(n=62) \text { batterers. } \\
\text { Hierarchical cluster analytic techniques } \\
\text { MANOVA among cluster batterers, } F(16,356)=34.93, p<.001 \text {, } \\
\eta^{2}=.61 \\
\text { Significant differences between batterer subgroups on PCL-R } \\
\text { Affective, } F(2,184)=16.97, p<.001 ; \eta^{2}=0.16 \text { : antisocial }> \\
\text { (non pathological }=\text { disturbed batterers), Lifestyle, } F(2,184) \\
=11.49, p<.001 ; \eta^{2}=0.11 \text { : (antisocial }=\text { disturbed) }>\text { non } \\
\text { pathological batterers, and Antisocial facet, } F(2,184)=26.65 \text {, } \\
p<.001 ; \eta^{2}=0.22 \text { : (antisocial = disturbed) }>\text { non pathological } \\
\text { batterers. } \\
\text { Not significant differences between batterer subgroups on } \\
\text { PCL-R Interpersonal facet, } F(2,184)=2.48, p=\text { n.s.; } \eta^{2}=0.03 \text {. } \\
\text { ANOVA }\end{array}$ \\
\hline
\end{tabular}


Table 4 (Continuation)

Empirical quantitative studies about the role of psychopathic traits on intimate partner violence perpetration among convicted male batterers $(n=21 ;$ $51 \%$ of the included studies)

\begin{tabular}{|c|c|c|c|}
\hline Paper & Sample & Assessment & Relevant findings \\
\hline $\begin{array}{l}\text { Echeburúa and Fernández- } \\
\text { Montalvo (2007) }\end{array}$ & $\begin{array}{l}\text { Prison sample }(N=162) \text { composed by } \\
\text { men sentenced for a serious IPV act } \\
\text { Non control group composed by non IPV } \\
\text { perpetrators } \\
100 \% \text { male } \\
\text { Range age: } 18-65 \text { years }\end{array}$ & $\begin{array}{l}\text { P: PCL-R } \\
\text { IPV: not assessed. } \\
\text { Convicted men }\end{array}$ & $\begin{array}{l}\text { Prevalence of psychopathy among male } \\
\text { batterers in prison: } 12.34 \% \\
\text { Role of psychopathic traits to establish } \\
\text { batterer typologies: } \\
\text { Batterers were classified as psychopaths } \\
(n=20 \text { ) and non psychopaths ( } n=142 \text { ) } \\
\text { based on a cut-off of } 20 \text { on PCL-R for } \\
\text { diagnostic of psychopathy. } \\
\text { Not significant differences between } \\
\text { psychopaths and non psychopaths } \\
\text { batterers on IPV homicide ( } \chi^{2}=.08, p \\
=.97 \text { ). } \\
\text { Group comparisons ( } t \text { and } \chi^{2} \text { test) }\end{array}$ \\
\hline Eckhardt et al. (2008) & $\begin{array}{l}\text { Forensic sample }(N=190) \\
\text { composed by men who were court- } \\
\text { referred to BIP } \\
\text { Non control group composed by non IPV } \\
\text { perpetrators } \\
100 \% \text { male } \\
\text { Mean age (SD): } 33.0(8.8)\end{array}$ & $\begin{array}{l}\text { P: SRP-II } \\
\text { IPV: CTS-2 physical, } \\
\text { sexual and } \\
\text { psychological } \\
\text { aggression and } \\
\text { injuries subscales, } \\
\text { MMEA }\end{array}$ & $\begin{array}{l}\text { Batterers were classified as high anger- } \\
\text { expressive }(n=56) \text {, moderate anger- } \\
\text { inexpressive }(n=13) \text {, and low anger }(n= \\
118) \text { groups, based on STAXI subscales. } \\
\text { Cluster analytic techniques } \\
\text { Role of psychopathic traits to differentiate } \\
\text { among batterer subtypes: } \\
\text { Significant differences between high } \\
\text { anger-expressive and low anger } \\
\text { batterers on SRP-II Total, } F(3,187)= \\
13.45, p<.01 \text {, and Factor } 2, F(3,187)= \\
13.13, p<.01 \text { : high anger }>\text { low anger. } \\
\text { Significant differences between batterer } \\
\text { clusters on SRP-II Factor } 1, F(3,187)= \\
2.78, p<.05 \text { : expressive batterers } \\
\text { displayed the highest score on Factor } 1 \\
\text { psychopathy. } \\
\text { ANOVA }\end{array}$ \\
\hline $\begin{array}{l}\text { Fernández-Montalvo and } \\
\text { Echeburúa (2008) }\end{array}$ & $\begin{array}{l}\text { Prison sample }(N=76) \text { composed by men } \\
\text { sentenced for a serious IPV incident } \\
\text { Non control group composed by non IPV } \\
\text { perpetrators } \\
100 \% \text { male } \\
\text { Mean age }(S D): 39.6(9.2)\end{array}$ & $\begin{array}{l}\text { P: PCL-R } \\
\text { IPV: not assessed. } \\
\text { Convicted men }\end{array}$ & $\begin{array}{l}\text { Prevalence of psychopathy among male } \\
\text { batterers in prison: } 14.47 \% \\
\text { Role of psychopathic traits to establish } \\
\text { batterer typologies: } \\
\text { Batterers were classified as psychopaths } \\
(n=11) \text { and non psychopaths ( } n=65 \text { ) } \\
\text { based on a cut-off of } 20 \text { on PCL-R for } \\
\text { diagnostic of psychopathy. } \\
\text { Not significant differences between } \\
\text { psychopathic and non psychopathic } \\
\text { batterers on domestic homicide, } \chi^{2}= \\
02, p=n \text {. s. } \\
\text { Group comparisons ( } \chi^{2} \text { test) }\end{array}$ \\
\hline Flournoy and Wilson (1991) & $\begin{array}{l}\text { Forensic sample }(N=56) \\
\text { composed by men who were court- } \\
\text { referred }(99 \%) \text { and self-referred }(1 \%) \text { to BIP } \\
\text { Non control group composed by non IPV } \\
\text { perpetrators } \\
100 \% \text { male } \\
\text { Mean age (SD): } 32.9(9.8)\end{array}$ & $\begin{array}{l}\text { P: MMPI psychopathic } \\
\text { deviate scales } \\
\text { IPV: not assessed. } \\
\text { Convicted men }\end{array}$ & $\begin{array}{l}\text { Prevalence of psychopathic deviate- } \\
\text { depression high-point pair profile: } 7 \% \\
\text { Prevalence of psychopathic deviate- } \\
\text { masculinity/feminity high point pair } \\
\text { profile: } 7 \% \\
\text { Batterers were classified based on MMPI } \\
\text { scales: elevated profile depicting a } \\
\text { psychopathic deviate-depression high- } \\
\text { point par }(n=25) \text {, and normal range } \\
\text { profile }(n=31) \text {. } \\
\text { Cluster analytic techniques }\end{array}$ \\
\hline
\end{tabular}


Table 4 (Continuation)

Empirical quantitative studies about the role of psychopathic traits on intimate partner violence perpetration among convicted male batterers $(n=21$; $51 \%$ of the included studies)

\begin{tabular}{|c|c|c|c|}
\hline Paper & Sample & Assessment & Relevant findings \\
\hline Gondolf and White (2001) & $\begin{array}{l}\text { Forensic sample }(N=840) \\
\text { composed by men who were court- } \\
\text { referred }(82 \%) \text { and self-referred }(12 \%) \text { to } \\
\text { BIP, and their female partner ( } 59 \%) \\
\text { Non control group composed by non IPV } \\
\text { perpetrators } \\
100 \% \text { male } \\
\text { Range age: } 18-65 \text { years }\end{array}$ & $\begin{array}{l}\text { P: MCMI-III } \\
\text { (combination of } \\
\text { scales) } \\
\text { IPV: CTS (female } \\
\text { partner's reports) } \\
\text { Convicted men }\end{array}$ & $\begin{array}{l}\text { Prevalence of primary psychopathy } \\
\text { disorder among male batterers in BIP ( } N \\
=580 \text { ): } 9 \% \text { (based on combination of } \\
\text { MCMI-III levels of personality disfunction } \\
\text { and scores on anxiety, antisocial, } \\
\text { sadistic, and narcissistic scales). and } \\
\text { Prevalence of secondary psychopathy } \\
\text { disorder among male batterers in BIP ( } N \\
=580 \text { ): } 11 \% \text { (based on combination of } \\
\text { MCMI-III levels of personality disfunction } \\
\text { and scores on anxiety, negativistic, } \\
\text { antisocial, and sadistic scales). } \\
\text { Batterers were classified as repeat } \\
(n=122), \text { once ( } n=68 \text { ), and none } \\
\text { reassaulters ( } n=394) \text {, based on } 15-\text {-montf } \\
\text { follow-up (from partner-report CTS). } \\
\text { Role of psychopathic traits to differentiate } \\
\text { among batterer subtypes: } \\
\text { Not significant differences between } \\
\text { reassault types on primary }(p=n \text {. } s \text {.) nor } \\
\text { secondary }(p=n \text {. s.) psychopathy. } \\
\text { Significant differences between reassault } \\
\text { types on psychopathic tendencies, } \chi^{2}(2) \\
=9.523, p<.01: \text { repeated }>\text { none }> \\
\text { once reassaulter batterers. } \\
\text { Group comparisons ( } \chi^{2} \text { test) }\end{array}$ \\
\hline Harris et al. (201 1) & $\begin{array}{l}\text { Forensic sample }(N=547) \text { composed by } \\
\text { men with a police record for IPV } \\
\text { Non control group composed by non IPV } \\
\text { perpetrators } \\
100 \% \text { male } \\
\text { Mean age }(S D): \mathrm{nr}\end{array}$ & $\begin{array}{l}\text { P: PCL-R (from file } \\
\text { records alone) } \\
\text { IPV: not assessed. } \\
\text { Convicted men }\end{array}$ & $\begin{array}{l}\text { Predictive power of psychopathic traits } \\
\text { on IPV perpetration: } \\
\text { Significant positive correlation between } \\
\text { total number of IPV and psychopathy ( } r \\
=.39, p<.05 \text { ). } \\
\text { Bivariate correlations } \\
\text { Significant main effect of psychopathy } \\
\text { on IPV perpetration ( } \beta=.33, p<.05 \text { ). } \\
\text { Multiple linear regression, controlled for } \\
\text { antisocial personality disorder, and total } \\
\text { injury caused in nondomestic assault }\end{array}$ \\
\hline Hilton et al. (2001) & $\begin{array}{l}\text { Forensic psychiatric sample }(N=508) \\
\text { from maximum-security psychiatric facility } \\
\text { - Target group: wife assaulters }(n=88) \\
\text { - Control group: other offenders }(n=420) \\
100 \% \text { male } \\
\text { Mean IPV perpetrators' age (SD): } 37.23 \\
(11.75)\end{array}$ & $\begin{array}{l}\text { P: PCL-R (from file } \\
\text { records alone) } \\
\text { IPV: not assessed. } \\
\text { Convicted men }\end{array}$ & $\begin{array}{l}\text { Predictive power of psychopathic traits } \\
\text { on IPV perpetration: } \\
\text { Significant differences between IPV } \\
\text { perpetrators and other offenders on } \\
\text { PCL-R Total score, } t=5.80, p<.001 \text { : } \\
\text { other offenders }>\text { IPV perpetrators } \\
\text { Group comparisons ( } t \text { test) }\end{array}$ \\
\hline Hilton et al. (2008) & $\begin{array}{l}\text { Forensic sample composed by men with } \\
\text { a police record for IPV: Sample } 1(n=303) \\
\text { + sample } 2(n=346) \\
\text { Not control group composed by non IPV } \\
\text { perpetrators } \\
100 \% \text { male } \\
\text { Mean sample 1's age (SD): } 35.5(10.1) \\
\text { Mean sample 2's age (SD): } 35.3(10.0)\end{array}$ & $\begin{array}{l}\text { P: PCL-R (from file } \\
\text { records alone) } \\
\text { IPV: police reports, } \\
\text { CTS2 severe IPV } \\
\text { subscale, SARA, DA, } \\
\text { DVSI. }\end{array}$ & $\begin{array}{l}\text { Predictive power of psychopathic traits } \\
\text { on IPV perpetration: } \\
\text { Significant correlation between } \\
\text { psychopathy and dichotomous IPV } \\
\text { recidivism (rsample } 1=.22 ; p<.001 \text {; } \\
\text { rtotal sample }=.29 ; p<.01 \text { ), number } \\
\text { of recidivistic IPV acts (rsample } 1=.28 \text {; } \\
p<.001 ; \text { rtotal sample }=.36 ; p<.01 \text { ), } \\
\text { total victim injury in recidivism (rsample } 1 \\
=.31 ; p<.001 ; \text { rtotal sample }=.37 ; p \\
<.01 \text { ), and number of CTS severe acts } \\
\text { (rsample } 1=.26 ; p<.001 ; \text { rtotal sample } \\
=.29 ; p<.01 \text { ). } \\
\text { Bivariate correlations }\end{array}$ \\
\hline
\end{tabular}


Table 4 (Continuation)

Empirical quantitative studies about the role of psychopathic traits on intimate partner violence perpetration among convicted male batterers $(n=21 ;$ $51 \%$ of the included studies)

\begin{tabular}{|c|c|c|c|}
\hline Paper & Sample & Assessment & Relevant findings \\
\hline Hornsveld et al. (2008) & $\begin{array}{l}\text { Forensic psychiatric sample } \\
\text { - Target group: domestically violent } \\
\text { outpatients }(n=63) \\
\text { - Control group: generally violent } \\
\text { outpatients }(n=103) \\
100 \% \text { Male } \\
\text { Mean domestically violent men's age } \\
\text { (SD): } 37.32(11.55)\end{array}$ & $\begin{array}{l}\text { P: PCL-R ( } 37.95 \% \text { from } \\
\text { file records alone) } \\
\text { IPV: not assessed. } \\
\text { Convicted men }\end{array}$ & $\begin{array}{l}\text { Predictive power of psychopathic traits } \\
\text { on IPV perpetration: } \\
\text { Not significant differences between } \\
\text { domestically and generally violent } \\
\text { outpatients on PCL-R Total, } F(2,163)= \\
2.75, p=n \text {. S., and Factor } 1, F(2,163)= \\
0.48, p=n \text {. s. } \\
\text { Significant differences between } \\
\text { domestically and generally violent on } \\
\text { PCL-R Factor } 2, F(2,163)=6.16, p<.004 \text { : } \\
\text { generally violent }>\text { domestically violent } \\
\text { outpatients } \\
\text { ANCOVA controlled for age }\end{array}$ \\
\hline $\begin{array}{l}\text { Huss and Langhinrichsen- } \\
\text { Rohling (2006) }\end{array}$ & $\begin{array}{l}\text { Forensic sample }(N=131) \\
\text { composed by men who were court- } \\
\text { referred }(59 \%) \text { and self-referred }(41 \%) \text { to } \\
\text { BIP } \\
\text { Non control group composed by non IPV } \\
\text { perpetrators } \\
100 \% \text { male } \\
\text { Mean age }(S D): 35.53(9.76)\end{array}$ & $\begin{array}{l}\text { P:PCL-SV (from } \\
\text { interview + file } \\
\text { records) } \\
\text { IPV: CTS-2 }\end{array}$ & 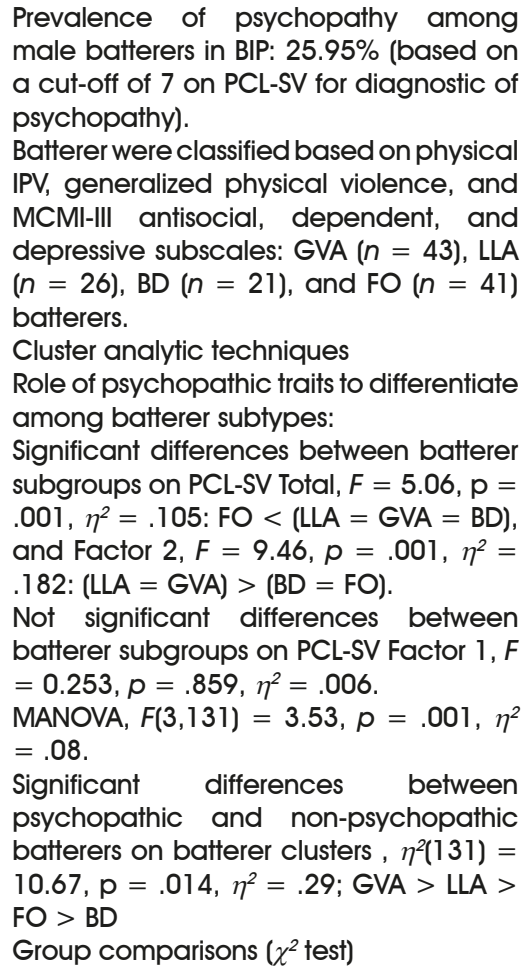 \\
\hline Juodis et al. (2014a) & $\begin{array}{l}\text { Prison sample } \\
\text { - Target group: domestic homicide } \\
\text { perpetrators }(n=37): 75.67 \% \text { of them } \\
\text { were IPV homicide perpetrators } \\
\text { - Control group: non-domestic homicide } \\
\text { perpetrators }(n=78) \\
100 \% \text { male } \\
\text { Mean IPV homicide perpetrators' age } \\
\text { (SD): nr }\end{array}$ & $\begin{array}{l}\text { P: PCL-R ( } 20.87 \% \text { from } \\
\text { file records alone) } \\
\text { IPV: DA }\end{array}$ & $\begin{array}{l}\text { Prevalence of psychopathy among } \\
\text { domestic homicide perpetrators in } \\
\text { prison: } 18.9 \% \text { (based on a cut-off of } 30 \\
\text { on PCL-R for diagnostic of psychopathy). } \\
\text { Predictive power of psychopathic traits } \\
\text { on IPV perpetration: } \\
\text { Significant differences between } \\
\text { domestic and non-domestic homicide } \\
\text { perpetrators on PCL Total, } t(113)=3.32 \text {, } \\
p \leq .01, d=0.64 \text {, Factor } 1, t(113)=2.12 \text {, } \\
p \leq .05 ; d=0.42 \text {, and Factor } 2, t(113)= \\
4.04, p \leq .001 ; d=0.76 \text { : in all cases, } \\
\text { non-domestic }>\text { domestic homicide } \\
\text { perpetrators. } \\
\text { Group comparison ( } t \text { test) }\end{array}$ \\
\hline
\end{tabular}


Table 4 (Continuation)

Empirical quantitative studies about the role of psychopathic traits on intimate partner violence perpetration among convicted male batterers $(n=21 ;$ $51 \%$ of the included studies)

\begin{tabular}{|c|c|c|c|}
\hline Paper & Sample & Assessment & Relevant findings \\
\hline Kalichman (1988) & $\begin{array}{l}\text { Forensic sample }(N=55) \\
\text { - Target group: intimate partner homicide } \\
\text { perpetrators ( } n=36 ; 55.55 \% \text { male) } \\
\text { - Control group: non-intimate partner } \\
\text { homicide men }(n=78) \\
71 \% \text { male } \\
\text { Mean IPV homicide men's age (SD): } 37(\mathrm{nr})\end{array}$ & $\begin{array}{l}\text { P: MMPI psychopathic } \\
\text { deviate scale } \\
\text { IPV: not assessed. } \\
\text { Convicted men }\end{array}$ & $\begin{array}{l}\text { RESULTS FOR MEN: } \\
\text { Prevalence of psychopathic deviate } \\
\text { among IPV homicide perpetrators: } \\
35 \% \text { (based on T score > } 70 \text { on MMPI } \\
\text { psychopathic deviate scale). } \\
\text { Predictive power of psychopathic traits } \\
\text { on IPV perpetration: } \\
\text { Not significant differences between IPV } \\
\text { and non-IPV homicide perpetrators on } \\
\text { psychopathy }(p=n \text {. } s .) \text {. } \\
\text { Group comparison }(t \text { test) }\end{array}$ \\
\hline Lawson et al. (2010) & $\begin{array}{l}\text { Forensic sample } \\
\text { - Target group: men on probation for IPV } \\
\text { ( } n=95 \text { ) } \\
\text { - Control group: men on probation for } \\
\text { non IPV ( } n=26) \\
100 \% \text { male } \\
\text { Mean IPV men's age (SD): } 31.0(8.5)\end{array}$ & $\begin{array}{l}\text { P: MMPI-2 } \\
\text { psychopathic deviant } \\
\text { scale } \\
\text { IPV: CTS physical } \\
\text { aggressionsscale }\end{array}$ & $\begin{array}{l}\text { Role of psychopathic traits to establish } \\
\text { batterer typologies: } \\
\text { Batterers were classified based on MMPI- } \\
2 \text { Hypochondriasis, } \\
\text { Depression, Hysteria, Psychopathic } \\
\text { Deviant, Paranoia, Psychoasthenia, } \\
\text { Schizophrenia, and Hypomania scales: } \\
\text { borderline ( } n=19 \text { ), antisocial ( } n=24 \text { ), } \\
\text { psychotic ( } n=26 \text { ) and nonpathological } \\
\text { ( } n=26 \text { ) batterers. } \\
\text { Cluster analytic techniques } \\
\text { All antisocialbatterers were characterized } \\
\text { by MMPI-2 profiles based on any } \\
\text { combination of psychopathic deviant } \\
\text { and other clinical scales: psychopathic } \\
\text { deviant ( } n=9 \text { ), the combination } \\
\text { between psychopathic deviant and } \\
\text { hypomania ( } n=6 \text { ), paranoia ( } n=3 \text { ), } \\
\text { hypocohondriasis ( } n=2 \text { ), depression ( } n \\
=1 \text { ), hysteria ( } n=1 \text { ), psychoasthenia ( } n \\
=1 \text { ), and schizophrenia ( } n=1 \text { ). } \\
\text { Borderline batterers were characterized } \\
\text { by MMPI-2 profiles based on combination } \\
\text { of schizophrenia, depression and } \\
\text { other clinical scales: } 84 \% \text { of borderline } \\
\text { batterers displayed any psychopathic } \\
\text { deviant symptoms. } \\
\text { Psychotic batterers were characterized } \\
\text { by MMPI-2 profiles based on combination } \\
\text { of hypomania, } \\
\text { paranoia and other clinical scales: } \\
\text { None of psychotic batterers displayed } \\
\text { psychopathic deviant symptoms. }\end{array}$ \\
\hline Murphy et al. (2007) & $\begin{array}{l}\text { Forensic sample }(N=139) \text { composed } \\
\text { by men who were court- }(85 \%) \text { and self- } \\
\text { referred (15\%) to BIP, and their female } \\
\text { partner }(n=104) \text { a } \\
\text { Non control group composed by non IPV } \\
\text { perpetrators } \\
100 \% \text { male } \\
\text { Mean age (SD): } 35.65(8.99)\end{array}$ & $\begin{array}{l}\text { P: SRP-II } \\
\text { IPV: CTS phyical } \\
\text { and psychological } \\
\text { aggression scales, } \\
\text { CTS2 injury scale, and } \\
\text { MMEA female } \\
\text { (self- and forther-reports) } \\
\text { partne }\end{array}$ & $\begin{array}{l}\text { Batterer were classifies based on STAXI } \\
\text { subscales: pathological anger }(n=26) \text {, } \\
\text { low anger control }(n=43) \text {, and normal } \\
\text { anger }(n=70) \text { batterers. } \\
\text { Cluster analytic techniques } \\
\text { Role of psychopathic traits to differentiate } \\
\text { among batterer subtypes: } \\
\text { Significant differences between batterer } \\
\text { subgroups on psychopathy, } F(2,119)= \\
4.48, p<.01 ; \eta^{2}=.07 \text { : pathological }> \\
\text { low }>\text { normal anger batterers. } \\
\text { ANOVA }\end{array}$ \\
\hline
\end{tabular}


Table 4 (Continuation)

Empirical quantitative studies about the role of psychopathic traits on intimate partner violence perpetration among convicted male batterers ( $n=21$; $51 \%$ of the included studies)

\begin{tabular}{|c|c|c|c|}
\hline Paper & Sample & Assessment & Relevant findings \\
\hline Rock et al. (2013) & $\begin{array}{l}\text { Forensic sample }(N=483) \text { composed by } \\
\text { men who were court-referred to BIP } \\
\text { Non control group composed by non IPV } \\
\text { perpetrators } \\
100 \% \text { male } \\
\text { Mean age }(S D): 35.14(10.16)\end{array}$ & $\begin{array}{l}\text { P: MMPI-2 } \\
\text { combination of scales } \\
\text { based on Sellbom et } \\
\text { al. (2012) } \\
\text { IPV: not assessed. } \\
\text { Convicted men }\end{array}$ & $\begin{array}{l}\text { Predictive power of psychopathic traits } \\
\text { on IPV perpetration: } \\
\text { Significant correlation between IPV } \\
\text { versatility and global psychopathy ( } r= \\
.19 ; p<.006) \text {, and impulsive/antisociality } \\
\text { traits }(r=.21 ; p<.006) \text {, but not fearless } \\
\text { dominance traits ( } r=.00 ; p=\text { n.s.). } \\
\text { Zero-Order correlations }\end{array}$ \\
\hline Stanford et al. (2008) & $\begin{array}{l}\text { Forensic sample }(N=113) \text { composed by } \\
\text { men who were court-referred to BIP } \\
\text { Non control group composed by non IPV } \\
\text { perpetrators } \\
100 \% \text { male } \\
\text { Mean age }(S D): \mathrm{nr}\end{array}$ & $\begin{array}{l}\text { P: PPI } \\
\text { IPV: not assessed. } \\
\text { Convicted men }\end{array}$ & $\begin{array}{l}\text { Batterers were classified as impulsive ( } n \\
=76) \text { and premeditated }(n=37) \text { based } \\
\text { on IPAS scores. } \\
\text { Role of psychopathic traits to differentiate } \\
\text { among batterer subtypes: } \\
\text { Significant differences between } \\
\text { impulsive and premeditated batterers on } \\
\text { PPI, } F(8,104)=4.99, p<.001 \text {; Wilk's } \Lambda= \\
0.72, \text { partial } \eta^{2}=.28 \text {. } \\
\text { MANOVA } \\
\text { Significant differences between impulsive } \\
\text { and premeditated batterers on PPI } \\
\text { Total score, } F(1,111)=11.19, p=.001 \text {, } \\
\text { partial } \eta^{2}=.09, \text { Fearless dominance } \\
\text { factor, } F(1,111)=6.01, p=.02, \text { partial } \\
\eta^{2}=.05, \text { and Impulsive/antisocial factor, } \\
F(1,111)=4.60, p=.03, \text { partial } \eta^{2}=.04 \text {, } \\
\text { Impulsive nonconformity scale, } F(1,111) \\
=17.90, p<.001, \text { partial } \eta^{2}=.14 \text {, } \\
\text { Stress inmunity scale, } F(1,111)=6.90, p \\
=.01, \text { partial } \eta^{2}=.06 \text {, and Fearlesness, } \\
F(1,111)=6.14, p=.02, \text { partial } \eta^{2}=.05 \text { : } \\
\text { in all cases, premeditated }>\text { impulsive } \\
\text { batterers. } \\
\text { Not significant differences between } \\
\text { impulsive and premeditated batterers on } \\
\text { PPI Blame externalization, Machiavellian } \\
\text { egocentrity, Carefree nonplanfulness, } \\
\text { Social potency, and Coldheartedness } \\
\text { scales }(p=n . s .) \text {. } \\
\text { ANOVA }\end{array}$ \\
\hline Swogger et al. (2007) & $\begin{array}{l}\text { Prison sample }(N=172) \\
\text { - Target group: antisocial batterers ( } n= \\
\text { 85) } \\
\text { - Control group: antisocial non-batterers } \\
(n=87) \\
\text { All inmates exhibited antisocial personality } \\
\text { features, based on DSM-IV-TR criteria for } \\
\text { APD. } \\
\text { Target and control group were created } \\
\text { based on review of criminal charges. } \\
\text { 100\% male } \\
\text { Mean target group's age (SD): } 28.42 \\
\text { (6.80) }\end{array}$ & $\begin{array}{l}\mathrm{P}: \mathrm{PCL}-\mathrm{R} \text { (from } \\
\text { interview }+ \text { file } \\
\text { records) } \\
\text { IPV: review of criminal } \\
\text { charges and self- } \\
\text { reported IPV }\end{array}$ & $\begin{array}{l}\text { Predictive power of psychopathic traits } \\
\text { on IPV perpetration: } \\
\text { Significant relationship between batterer } \\
\text { vs. non-batterer group membership and } \\
P C L-R \text { Affective }(B=0.29, S E=0.11, p< \\
\left..05, O R=1.34, R^{2}=0.06\right) \text { and Lifestyle } \\
\text { facets ( } B=-0.24, S E=0.11, p<.05, O R \\
\left.=0.79, R^{2}=0.04\right) \text {. } \\
\text { Not significant relationship between } \\
\text { batterer vs. non-batterer group } \\
\text { membership and PCL-R Total ( } B=0.03 \text {, } \\
S E=0.03, p>.05 \text { ), Interpersonal ( } B= \\
-0.04, S E=0.10, p>.05), \text { and Antisocial } \\
\text { facets ( } B=0.13, S E=0.10, p>.05 \text { ). } \\
\text { Results of the analyses including self- } \\
\text { reports of IPV indicated a pattern of } \\
\text { results equivalent to those presented } \\
\text { previously. } \\
\text { Logistic regression analyses, controlled } \\
\text { for other violent charges }\end{array}$ \\
\hline
\end{tabular}


Table 4 (Continuation)

Empirical quantitative studies about the role of psychopathic traits on intimate partner violence perpetration among convicted male batterers $(n=21$; $51 \%$ of the included studies)

\begin{tabular}{|c|c|c|c|}
\hline Paper & Sample & Assessment & Relevant findings \\
\hline Swogger et al. (2012) & $\begin{array}{l}\text { Forensic sample }(N=75) \text { composed by } \\
\text { men on probation } \\
\text { Not control group composed by non IPV } \\
\text { perpetrators } \\
100 \% \text { male } \\
\text { Mean age }(S D): 34(11.4)\end{array}$ & $\begin{array}{l}\text { P: PCL-R (from } \\
\text { interview + file } \\
\text { records) } \\
\text { IPV: "ad hoc" } \\
\text { assessment tool }\end{array}$ & 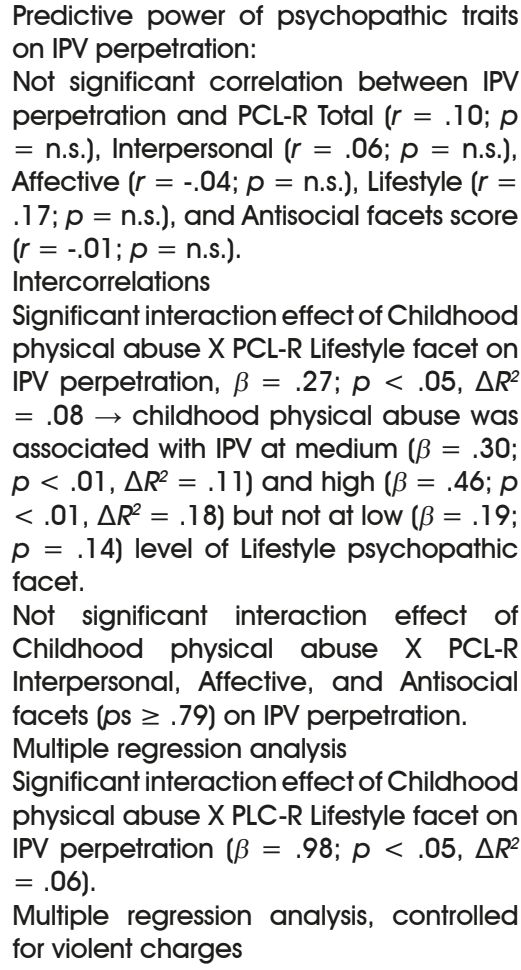 \\
\hline Taft et al. (2004) & $\begin{array}{l}\text { Forensic sample }(N=107) \text { composed } \\
\text { by men who were court- }(88 \%) \text { or self- } \\
\text { referred }(12 \%) \text { to BIP } \\
\text { Non control group composed by non IPV } \\
\text { perpetrators } \\
100 \% \text { male } \\
\text { Mean age }(S D): \mathrm{nr}\end{array}$ & $\begin{array}{l}\text { P: SRP-II } \\
\text { IPV: all participants } \\
\text { had a documented } \\
\text { problem with IPV }\end{array}$ & $\begin{array}{l}\text { Predictive power of psychopathic traits } \\
\text { on IPV perpetration: } \\
\text { Significant correlation between } \\
\text { psychopathy and number of arrests for } \\
\text { IPV }(r=.25 ; p<.05) \text {. } \\
\text { Intercorrelations }\end{array}$ \\
\hline \multicolumn{4}{|c|}{ 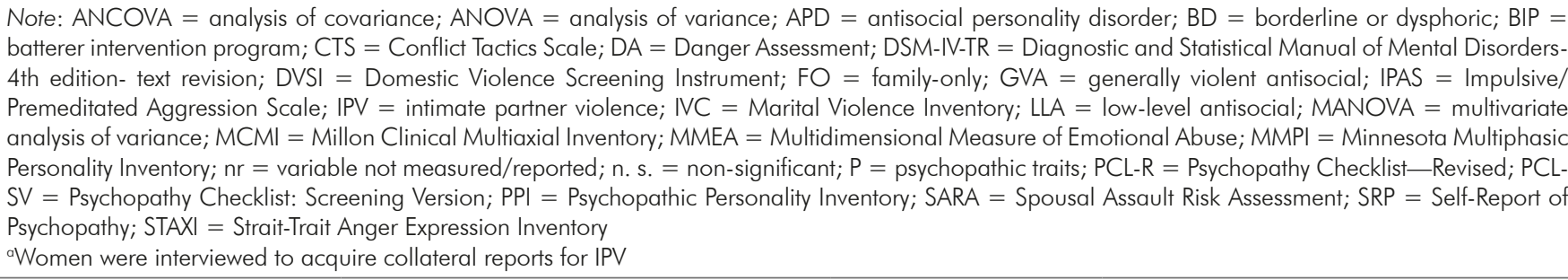 } \\
\hline
\end{tabular}

Gottman et al. (1995). Second, psychopathy was not a useful variable to uniquely differentiate GVA batterers, as Holtzworth-Munroe and Stuart (1994) had hypothesized: significant differences between GVA, borderline, and low-level antisocial batterers on psychopathy were not found, and GVA batterers were only more psychopathic than family-only (FO) batterers (Holtzworth-Munroe et al., 2000, 2003). Alternative batterer typologies established dependent upon the severity of IPV perpetration evidenced that psychopathy was not associated with a greater severity of IPV perpetration, because significant differences in psychopathy between severely and low-level violent batterers were not found (Babcock et al., 2005). Instead, when the batterer typology was established dependent on the generalization of violent behavior outside partner relationshipsFO (only IPV perpetrators) vs. generally violent (both inside and outside the home) batterers- 
psychopathy was useful to differentiate $\mathrm{FO}$ from generally violent: the latter displayed the highest levels of psychopathy (Theobald et al., 2015). Finally, when the batterer typology was established dependent on the type of IPV perpetrated (proactive or reactive), findings evidenced that proactive partner-violent men were more psychopathic than reactive partnerviolent men (Chase et al., 2001).

Unlike the studies presented above, three studies in which the core psychopathic traits were entered into cluster analyses to establish batterer typologies were identified. First, Babcock et al. (2008) tested the batterer typology proposed by Holtzworth-Munroe and Stuart (1994), including the SRP Factor 1 instead of antisocial traits into the cluster analysis to avoid the empirical and conceptual overlap between borderline and antisocial personality disorders. However, Babcock et al. (2008) also failed to identify uniquely GVA batterers, who displayed significantly higher levels of core psychopathic traits than borderline but not than $\mathrm{FO}$ batterers. Second, Walsh et al. (2010) established a batterer typology based on personality features, including the PCL-SV Facets 1 to 3 into the cluster construction (the Antisocial facet was excluded because it reflects behavioral rather than personality traits). In this way, psychopathy was successful in detecting a specific batterer subgroup, labeled antisocial batterers. Finally, Hale et al. (1988), based on psychopathology batterer typology, detected a specific batterer subtype characterized by a combination of psychopathic deviate and depression.

\section{-EVIDENCE FROM STUDIES CONDUCTED WITH CONVICTED MALE BATTERERS}

Again, psychopathy was commonly used to examine the differences among batterer subtypes. Unlike studies testing the HoltzworthMunroe and Stuart (1994) batterer typology among non-convicted samples, Huss and Langhinrichsen-Rohling (2006) evidenced that psychopathy seems to be a useful variable to identify uniquely GVA batterers among a forensic sample. However, more exhaustive analysis of these results revealed that batterer subtypes did not differ in the core psychopathic traits (PCLSV Factor 1), but GVA and low-level antisocial batterers displayed higher scores on behavioral psychopathic traits than other batterer subtypes. When other alternative batterer typologies were established, psychopathy proved to be useful to identify a specific batterer subtype. Psychopathic batterers belonged to batterer subtypes characterized by pathologically high anger levels (Eckhardt et al., 2008; Murphy et al., 2007), premeditated IPV perpetration (Stanford et al., 2008), and persistent IPV recidivism (Gondolf \& White, 2001).

Unlike the studies presented above, four studies in which psychopathy was entered into cluster analyses to establish batterer typologies were identified. First, based on three dimensions suggested by Holtzworth-Munroe and Stuart (1994), Cunha and Gonçalves (2013) carried out cluster analyses including both affective and antisocial psychopathic traits and established three batterer subtypes. The Affective but not Antisocial facet was useful in detecting a specific batterer subtype: the so-called antisocial batterers displayed higher affective psychopathic traits than the other batterer subtypes. Second, other batterer typologies based on psychopathology (the MMPI-2 psychopathic deviate scale was entered into the cluster analysis) were proposed by Lawson et al. (2010), who distinguished between antisocial, borderline, psychotic and non-pathological batterers. Psychopathy failed to detect a specific batterer subtype: profiles characterized by psychopathic deviate were found among antisocial and borderline batterers. Finally, when batterers were classified based on a categorical approach to psychopathy, psychopathic batterers were not more likely to perpetrate intimate partner homicide than were non-psychopathic batterers (Echeburúa \& Fernández-Montalvo, 2007; FernándezMontalvo \& Echeburúa, 2008).

\section{DISCUSSION}

The purpose of this systematic review was to examine the role of psychopathic traits in male-to-female IPV perpetration. To this end, forty-one studies from PsycINFO, Scopus and WoS databases were analyzed and classified according the aim of the research (the analysis of the predictive power of psychopathy on IPV perpetration vs. of the usefulness of psychopathy to differentiate among batterer subtypes), the type of sample used (non-convicted partner- 
violent men vs. convicted male batterers) and the psychopathy measurement approach (including vs. excluding antisocial traits as diagnostic criteria).

Regarding the predictive power of psychopathy on IPV perpetration, the majority of studies supported the role of psychopathy as a robust predictor of male-to-female IPV perpetration. Studies underscoring the predictive power of both global psychopathy (including antisocial traits; Kiire, 2017; Okano et al., 2016) and the core psychopathic features (Bates et al., 2017; Coyne et al., 2010; Mager et al., 2014; Marshall \& Holtzworth-Munroe, 2010; Wymbs et al., 2017) on IPV perpetration were identified. However, further research separately analyzing the core personality and the behavioral psychopathic traits is necessary to clarify the potential differential relationship of interpersonal/affective traits with physical and psychological IPV perpetration, suggested in opposite directions by Coyne et al. (2010) and Wymbs et al. (2017). The predictive power of psychopathy was also examined comparing partner-violent men with non-IPV men. Among non-convicted samples, psychopathy did not predict IPV perpetration (partner-violent men displayed as many psychopathic traits as non-IPV men; Babcock et al., 2005, 2008; Holtzworth-Munroe et al., 2000; Walsh et al., 2010). However, IPV men were initially more generally violent than non-IPV men, so these results could be biased by the strong association between psychopathy and violence historically documented in the literature (Hecht et al., 2016; Reidy et al., 2015) and not reflect the specific relationship between psychopathy and violent behavior against a partner. Instead, among convicted samples matched in their violence level (namely, batterers compared with other violent offenders), the results suggested that batterers displayed as many core psychopathic features as the other violent offenders (Hornsveld et al., 2008) but fewer behavioral psychopathic traits than the other violent offenders (Hornsveld et al., 2008; Swogger et al., 2007). These results suggested an image of batterers consistent with the subclinical manifestation of psychopathy already proposed by Cleckley (1941). The adaptive lifestyle that characterizes the successful psychopath (Hall \& Benning, 2006) would allow the psychopath batterer to mask his emotional coldness and lack of empathy and blame, to limit his violent behavior inside the home (Pérez, Rodríguez-Díaz, Herrero, \& Fernández-Suárez, 2016), and to be involved in subtle forms of IPV characterized by manipulation and control of their partners (Coyne et al., 2010). Further research matching batterers and non-batterers in their generalized violence level and separately examining each psychopathic trait will be necessary to confirm the hypothesis of batterer as successful psychopathy. If this hypothesis were correct, BIPs aimed at reducing impulsivity and irresponsible behavior might be not effective, and interventions designed to increase empathy would be necessary (Swogger et al., 2007). However, to date, effective treatments aimed at developing empathic responses among psychopaths have not been identified (Roche, Shoss, Pincus, \& Ménard, 2011; Swogger et al., 2007). Moreover, further studies measuring psychopathy correctly and statistically controlling antisocial and generalized violence, as Swogger et al. (2007), which would be necessary to confirm the specific predictive power of affective psychopathic traits on IPV perpetration suggested by Cunha and Gonçalves (2013), and Swogger et al. (2007).

Regarding the utility of psychopathic traits in batterer typologies, some researchers used psychopathy to establish batterer typologies, and others used it to differentiate among previously established batterer subtypes. When psychopathy was used to examine the differentiate among batterer subtypes, psychopathic traits failed to differentiate among traditional batterer subtypes proposed by Gottman et al. (1995) and Holtzworth-Munroe and Stuart (1994), among both non-convicted (Babcock et al., 2004; Holtzworth-Munroe et al., 2000, 2003; Meehan et al., 2001) and convicted (Huss \& Langhinrichsen-Rohling, 2006) samples. However, when other alternative batterer typologies were established, psychopathy proved to be useful to identify a specific batterer subtype characterized by displaying pathologically high anger levels (Eckhardt et al., 2008; Murphy et al., 2007), perpetrating premeditated (Stanford et al., 2008) and proactive IPV (Chase et al., 2001) and repeatedly reassaulting their partners (Gondolf \& White, 2001). The relationship between the core psychopathic traits and difficulty controlling anger, as well as the tendency toward 
premeditated and proactive violence, was consistent with previous literature (Bo, Abu-Akel, Kongerslev, Haahr, \& Bateman, 2014; Hecht et al., 2016; Myers \& Monaco, 2000). Precisely, these batterer subtypes that displayed higher levels of psychopathy were responsible for the highest levels of psychological and physical IPV (Eckhardt et al., 2008), even across time (Murphy et al., 2007), and were more likely to reject treatment (Stanford et al., 2008) and persistently recidivate to IPV (Eckhardt et al., 2008). So, psychopathy was able, indirectly, to detect the most frequent batterer subtype. Therefore, psychopathy should be especially considered among batterers who display high levels of anger, premeditated and proactive IPV and persistent IPV recidivism. Generalized violence seems to be a key variable to differentiate among batterers in terms of psychopathic traits. As evidenced by Theobald et al. (2015), who revealed that specialist batterers - men who are violent only inside intimate partner relationships-achieved significantly lower scores on psychopathy than generalist batterers - men who are also violent outside intimate partner relationships. The image of the batterer as successful psychopath suggested above could be more coherent with specialist batterers than with generalist batterers (similar to other violent offenders, as seen in Theobald et al., 2015). Although Theobald et al. (2015) did not confirm the hypothesis about the specialist batterer with higher affective traits than the generalist batterer, it is possible that the erroneous psychopathy measurement by Theobald et al. (2015), conducted without the face-to-face interview, has underestimated affective psychopathic traits. Thus, further batterer typology studies based on generalization of violence, as in Herrero, Torres, FernándezSuárez, and Rodríguez-Díaz (2016) or Theobald et al. (2015), are recommended.

Finally, although psychopathy was able to detect the most frequent batterer subtype, psychopathy did not differ between batterer typologies based on severity of IPV perpetration among both non-convicted (Babcock et al., 2005) and convicted samples (Echeburúa \& FernándezMontalvo, 2007; Fernández-Montalvo \& Echeburúa, 2008). Studies comparing nonlethal with lethal IPV perpetrators would help to examine the predictive power of psychopathy on severity IPV.

\section{- LIMITATIONS}

Contradictory findings detected across this paper could be due to methodological differences between reviewed studies. First, the conceptual confusion around the psychopathy construct was also reflected in IPV field research: 85\% of reviewed studies included antisocial traits as diagnostic criteria to assess psychopathy; however, recent researchers have begun to exclude items related to antisociality/criminality from psychopathy assessment tools or to statistically control the influence of antisocial features in their analyses. The inclusion of diagnostic criteria related to prior violence, like in PCL-R and its derivative scales, could contaminate the relationship between psychopathy and violence (Salvador, Pérez, Fernández, Bringas, \& Rodríguez-Díaz, 2015; Skeem, Poythress, Edens, Lilienfeld, \& Cale, 2003). As recommended by contemporary authors (Evans \& Tully, 2016; Skeem et al., 2003), future research should use psychopathy assessment tools that have excluded criminal/ antisocial behavior as diagnostic criteria or should statistically control antisocial traits. Moreover, although PCL-R and PCL-SV application requires the combination of information from interviews and file records, only $26 \%$ of reviewed studies (Huss and Langhinrichsen-Rohling, 2006; Mager et al., 2014; Swogger et al., 2007, 2012) followed these recommendations. Research in which PCL-R and PCL-SV are scored without both sources of information runs the risk of losing information about the individual's interpersonal style (Hare, 2016) or being rated based on manipulated information from perpetrators' selfreports. Thus, the review of collateral information in conjunction with the semi-structured interview is recommended for future studies measuring psychopathy through the PCL-R and the PCLSV. Additionally, few studies used a categorical approach of psychopathy, probably due to the low prevalence of psychopathy among community sample (Hare, 2003). It has prevented a comparative analyses between findings from the categorical and dimensional approaches. Future research should be addressed the differences between such approaches of psychopathy assessment and their implications.

Second, different criteria to categorize men as IPV perpetrators among community samples were used. IPV researchers should agree on how 
many IPV acts are necessary to consider men as partner-violent. "Zero tolerance" ideology (Sawyer, Coles, Williams, \& Williams, 2015), according to which no IPV act is acceptable (regardless of its frequency and severity), is recommended to identify partner-violent men among non-convicted samples.

Third, batterers and non-batterers were not equivalent regarding their generalized violence levels, so the results could actually reflect the association between psychopathy and general violence but not specific IPV. The inclusion of a control group that exhibits the same levels of generalized violence as batterers or the statistical control of generalized violence is recommended among both convicted and non-convicted samples.

Finally, the risk of bias of studies included in this review has not been evaluated. Future research should be assess the quality and risk of bias of studies included, as recommended by Moher et al. (2009) and Perestelo-Pérez (2013). However, bibliographical records included in databases used in this systematic review are reviewed by experts who ensure its high level of quality. Also, hand-searching was practiced in order to minimize bias (Vassar et al., 2016).

\section{- IMPLICATIONS}

This systematic review suggests several implications for future research and clinical practice. First, male batterers are not a homogeneous group in terms of their psychopathic traits. Generalized violence seems to be a key variable to differentiate among batterers in terms of psychopathic traits: unlike the historically documented GVA/generalist batterer (with high levels of both personality and behavioral psychopathic traits), another batterer subtype who display high score on personality but not on behavioral psychopathic traits was suggested (specialist/FO batterer). While generalist batterers could be consistent with traditional psychopaths (psychopathic group proposed by Hare. 2016), specialist batterers could be consistent with successful psychopaths (callousconning group proposed by Hare, 2016). In turn, while generalist batterers could benefit from conventional BIPs, specialist batterers might require specific BIPs targeted to the personality but not to behavioral psychopathic traits. An accurate differential diagnostic among batterers (non-psychopath, successful psychopath, and traditional psychopath) would help when designing effective, specific BIPs according their psychopathic traits. Second, psychopathy seems to be a useful variable to predict the frequency of IPV (and persistent recidivism) but not to predict IPV severity. Further research is necessary to highlight the role of psychopathic traits in IPV risk assessment.

\section{-Acknowledgements}

This work was supported by the Spanish Ministry of Economy and Competitiveness (MINECO-17-PSI2016-77484-P), the Foundation for the Promotion of Applied Scientific Research and Technology in Asturias (Severo Ochoa scholarships BP13134 and BP14-153), and the Spanish Ministry of Education, Culture and Sport (pre-doctoral contract at the Teacher Training University FPU 13/04310).

\section{- Conflict of interest}

The authors declare no conflict of interest.

\section{REFERENCES}

References marked with an asterisk indicate studies included in the systematic review.

Aaron, S. M., \& Beaulaurier, R. L. (2016). The need for new emphasis on batterers intervention programs. Trauma, Violence, \& Abuse, 18, 425-432. https://doi. org/10.1177/1524838015622440

Andershed, H., Kerr, M., Stattin, H., \& Levander, S. (2002). Psychopathic traits in non-referred youths: A new assessment tool. In E. Blauw \& L. Sheridan (Eds.), Psychopaths: Current international perspectives (pp. 131-158). The Hague: Elservier.

Arias, E., Arce, R., \& Vilariño, M. (2013). Batterer intervention programmes: A metaanalytic review of effectiveness. Psychosocial Intervention, 22, 153-160. https://doi. org/10.5093/in2013a18

Babcock, J. C., Green, C. E., \& Robie, C. (2004). Does batterers' treatment work? A metaanalytic review of domestic violence treatment. Clinical Psychology Review, 23, 1023-1053. https://doi.org/10.1016/i.cpr.2002.07.001

*Babcock, J. C., Green, C. E., \& Webb, S. A. (2008). Decoding deficits of different types of batterers during presentation of facial affect 
slides. Journal of Family Violence, 23, 295 302. https://doi.org/10.1007/s10896-0089151-1

*Babcock, J. C., Green, C. E., Webb, S. A., \& Graham, K. H. (2004). A second failure to replicate the Gottman et al. (1995) typology of men who abuse intimate partners... and possible reasons why. Journal of Family Psychology, 18, 396-400. https://doi. org/10.1037/0893-3200.18.2.396

*Babcock, J. C., Green, C. E., Webb, S. A., \& Yerington, T. P. (2005). Psychophysiological profiles of batterers: Autonomic emotional reactivity as it predicts the antisocial spectrum of behavior among intimate partner abusers. Journal of Abnormal Psychology, 114, 444-455. https://doi.org/10.1037/0021843X.1 14.3.444

*Bates, E. A., Archer, J., \& Graham-Kevan, N. (2017). Do the same risk and protective factors influence aggression toward partners and same-sex others? Aggressive Behavior, 43, 163-175. https://doi.org/10.1002/ ab.21672

*Belfrage, H., \& Rying, M. (2004). Characteristics of spousal homicide perpetrators: A study of all cases of spousal homicide in Sweden 19901999. Criminal Behaviour and Mental Health, 14, 121-133. https://doi.org/10.1002/ $\mathrm{cbm} .577$

Bo, S., Abu-Akel, A., Kongerslev, M., Haahr, U. H., \& Bateman, A. (2014). Mentalizing mediates the relationship between psychopathy and type of aggression in schizophrenia. Journal of Nervous and Mental Disease, 202, 55-63. https://doi.org/10.1097/ NMD.0000000000000067

Burt, G. N., Olver, M. E., \& Wong, S. C. P. (2016). Investigating characteristics of the nonrecidivating psychopathic offender. Criminal Justice and Behavior, 43, 1741-1760. https://doi. org/10.1177/0093854816661215

Carbajosa, P., Boira, S., \& Tomás-Aragonés, L. (2013). Difficulties, skills and therapy strategies in interventions with court-ordered batterers in Spain. Aggression and Violent Behavior, 18, 118-124. https://doi.org/10.1016/i. avb.2012.11.005

Carbajosa, P., Catalá-Miñana, A., Lila, M., \& Gracia, E. (2017). Differences in treatment adherence, programa completion, and recidivism among batterer subtypes. The European Journal of Psychology Applied to Legal Context, 9, 93-101. https://doi. org/10.1016/i.eipal.2017.04.001

*Chase, K. A., O'Leary, D., \& Heyman, R. E. (2001). Categorizing partner-violent men within the reactive-proactive typology model. Journal of Consulting and Clinical Psychology, 69, 567-572. https://doi. org/10.1037//0022-006X.69.3.567

Cleckley, H. (1941). The mask of sanity (1 st ed.). St. Louis: C. V. Mosby Company

Cooke, D. J., Michie, C., \& Skeem, J. (2007). Understanding the structure of the Psychopathy Checklist - Revised: An exploration of methodological confusion. British Journal of Psychiatry, 190, s39-s50. https://doi. org/10.1192/bip.190.5.s39

*Coyne, S. M., Nelson, D. A., Graham-Kevan, N., Keister, E., \& Grant, D. M. (2010). Mean on the screen: Psychopathy, relationship aggression, and aggression in the media. Personality and Individual Differences, 48, 288-293. https:// doi.org/10.1016/i.paid.2009.10.018

*Cunha, O., \& Gonçalves, R. A. (2013). Intimate partnerviolence offenders: Generating a databased typology of batterers and implications for treatment. The European Journal of Psychology Applied to Legal Context, 5, 131 139. https://doi.org/10.5093/ejpalc2013a2

Dargis, M. A., Mattern, A. C., \& Newman, J. P. (2017). Set-congruent priming stimuli normalize the information processing of psychopathic offenders. Journal of Psychopathology and Behavioral Assessment, 39, 209-219. https://doi.org/10.1007/ s10862-017-9587-9

Dil, S., \& Kazmi, F. (2016). Psychopathic inclination among incarcerated youth of Hazara Division Pakistan. SAGE Open, 6. https://doi. org/10.1177/2158244016671558

*Echeburúa, E., \& Fernández-Montalvo, J. (2007). Male batterers with and without psychopathy: An exploratory study in Spanish prisons. International Journal of Offender Therapy and Comparative Criminology, 51, 254-263. https://doi. org/10.1177/0306624X06291460

*Eckhardt, C. I., Samper, R. E., \& Murphy, C. M. (2008). Anger disturbances among perpetrators of intimate partner violence. Clinical characteristics and outcomes of court- 
mandated treatment. Journal of Interpersonal Violence, 23, 1600-1617. https://doi. org/10.1177/0886260508314322

Evans, L., \& Tully, R. J. (2016). The Triarchic Psychopathy Measure (TriPM): Alternative to the PCL-R? Aggression and Violent Behavior, 27, 79-86. https://doi.org/10.1016/i. avb.2016.03.004

Feder, L., \& Wilson, D. B. (2005). A metaanalytic review of court-mandated batterer intervention programs: Can courts affect abusers' behavior? Journal of Experimental Criminology, 1, 239-262. https://doi. org/10.1007/s $11292-005-1179-0$

Felthous, A. R. (2015). The appropriateness of treating psychopathic disorders. CNS Spectrums, 20, 182-189. https://doi. org/10.1017/S1092852915000243

*Fernández-Montalvo, J., \& Echeburúa, E. (2008). Trastornos de personalidad y psicopatía en hombres condenados por violencia grave contra la pareja. Psicothema, 20(2), 193196. Retrieved from http://sociales.redalyc. org/articulo.oa? id $=72720203$

*Flournoy, P. S., \& Wilson, G. L. (1991). Assessment of MMPI profiles of male batterers. Violence and Victims, 6(4), 309-320.

*Gondolf, E. W., \& White, R. J. (2001). Batterer program participants who repeatedly reassault. Psychopathic tendencies and other disorders. Journal of Interpersonal Violence, 16, 361-380. https://doi. org/10.1177/088626001016004006

Gottman, J. M., Jacobson, N. S., Rushe, R. H., Shortt, J. W., Babcock, J. C., LaTaillade, J. J., \& Waltz, J. (1995). The relationship between heart rate reactivity, emotionally aggressive behavior, and general violence in batterers. Journal of Family Psychology, 9, 227-248. https://doi.org/10.1037//08933200.9.3.227

*Hale, G., Zimostrad, S., Duckworth, J., \& Nicholas, D. (1988). Abusive partners: MMPI profiles of male batterers. Journal of Mental Health Counseling, 10(4), 214-224.

Hall, J. R., \& Benning, S. D. (2006). The "Successful" psychopath: Adaptive and subclinical manifestation of psyhopathy in the general population. In C. J. Patrick (Ed.), Handbook of psychopathy (pp. 459 - 480). New York, NY: The Guildford Press.

Hare, R. D. (2003). Manual for the Revised
Psychopathy Checklist (2a ed.). Toronto, ON, Canada: Multi-Health Systems.

Hare, R. D. (2016). Psychopathy, the PCL-R, and criminal justice: Some new findings and current issues. Canadian Psychology, 57, 21 34. https://doi.org/10.1037/cap0000041

*Harris, G. T., Hilton, N. Z., \& Rice, M. E. (2011). Explaining the frequency of intimate parner violence by male perpetrators. Do attitude, relationship, and neighborhood variables add to antisociality? Criminal Justice and Behavior, 38, 309-331. https://doi. org/10.1177/0093854810397449

Hart. S. D., Cox, D. N., \& Hare, R. D. (1995). Manual for the Psychopathy Checklist: Screening version (PCL:SV). Toronto, ON: Multi-Health Systems.

Hecht, L. K., Berg, J. M., Lilienfeld, S. O., \& Latzman, R. D. (2016). Parsing the heterogeneity of psychopathy and aggression: Differential associations across dimensions and gender. Personality Disorders: Theory, Research, and Treatment, 7, 2-14. https:// doi.org/10.1037/per0000128

Herrero, J., Torres, A., Fernández-Suárez, A., \& Rodríguez-Díaz, F. J. (2016). Generalist versus specialist: Toward a typology of batterers in prison. The European Journal of Psychology Applied to Legal Context, 8, 19-26. https:// doi.org/10.1016/i.ejpal.2015.09.002

*Hilton, N. Z., Harris, G. T., \& Rice, M. E. (2001). Predicting violence by serious wife assaulters. Journal of Interpersonal Violence, 16, 408-423. https://doi. org/10.1177/088626001016005002

*Hilton, N. Z., Harris, G. T., Rice, M. E., Houghton, R. E., \& Eke, A. W. (2008). An indepth actuarial assessment for wife assault recidivism: The Domestic Violence Risk Appraisal Guide. Law and Human Behavior, 32, 150-163. https:// doi.org/10.1007/s 10979-007-9088-6

* Holtzworth-Munroe, A., Meehan, J. C., Herron, K., Rehman, U., \& Stuart, G. L. (2000). Testing the Holtzworth-Munroe and Stuart (1994) batterer typology. Journal of Consulting and Clinical Psychology, 68, 1000-1019. https:// doi.org/10.1037//0022-006X.68.6.1000

*Holtzworth-Munroe, A., Meehan, J. C., Herron, K., Rehman, U., \& Stuart, G. L. (2003). Do subtypes of maritally violent men continue to differ over time? Journal of Consulting and Clinical Psychology, 71, 728-740. https:// 
doi.org/10.1037/0022-006X.71.4.728

Holtzworth-Munroe, A., \& Stuart, G. L. (1994). Typologies of male batterers: Three subtypes and the differences among them. Psychological Bulletin, 1 16, 476-497. https:// doi.org/10.1037/0033-2909.1 16.3.476

*Hornsveld, R. H. J., Bezuijen, S., Leenaars, E. E. M., \& Kraaimaat, F. W. (2008). Domestically and generally violent forensic psychiatric outpatients: Personality traits and behavior. Journal of Interpersonal Violence, 23, 1380-1393. https://doi. org/10.1177/0886260508314303

Huss, M. T., Covell, C. N., \& LanghinrichsenRohling, J. (2006). Clinical implications for the assessment and treatment of antisocial and psychopathic domestic violence perpetrators. Journal of Aggression, Maltreatment \& Trauma, 13, 59-85. https://doi.org/10.1300/ J146v13n01 04

Huss, M. T., \& Langhinrichsen-Rohling, J. (2000). Identification of the psychopathic batterer: The clinical, legal, and policy implications. Aggression and Violent Behavior, 5, 403422. https://doi.org/10.1016/S13591789(98)00038-X

*Huss, M. T., \& Langhinrichsen-Rohling, J. (2006). Assessing the generalization of psychopathy in a clinical sample of domestic violence perpetrators. Law and Human Behavior, 30, 571-586. https://doi.org/10.1007/s10979006-9052-x

*lyican, S., Sommer, J. M., Kini, S., \& Babcock, J. C. (2015). Collateral report of psychopathy: Convergent and divergent validity of the Psychopathic Personality Inventory-Short Form. Journal of Forensic Psychiatry and Psychology, 26, 476-492. https://doi.org/10.1080/147 89949.2015.1018926

Jacobson, N. S., \& Gottman, J. M. (1998). When men batter women: New insights into ending abusive relationships. New York: Simon \& Schuster.

*Juodis, M., Starzomski, A., Porter, S., \& Woodworth, M. (2014a). A comparison of domestic and non-domestic homicides: Further evidence for distinct dynamics and heterogeneity of domestic homicide perpetrators. Journal of Family Violence, 29, 299-313. https://doi.org/10.1007/s10896014-9583-8

Juodis, M., Starzomski, A., Porter, S., \&
Woodworth, M. (2014b). What can be done about high-risk perpetrators of domestic violence? Journal of Family Violence, 29, 381 -390. https://doi.org/10.1007/s10896014-9597-2

*Kalichman, S. C. (1988). MMPI profiles of women and men convicted of domestic homicide. Journal of Clinical Psychology, 44(6), 847-853.

*Kiire, S. (2017). Psychopathy rather than Machiavellianism or narcissism facilitates intimate partner violence via fast life strategy. Personality and Individual Differences, 104, 401-406. https://doi.org/10.1016/i. paid.2016.08.043

*Lawson, D. M., Brossart, D. F., \& Shefferman, L. W. (2010). Assessing gender role of partnerviolent men using the Minnesota Multiphasic Personality Inventory-2 (MMPI-2): Comparing abuser types. Professional Psychology: Research and Practice, 41, 260-266. https:// doi.org/10.1037/a0019589

Lilienfeld, S. O., \&Hess, T. H. (2001). Psychopathic personality traits and somatization: Sex differences and the mediating role of negative emotionality. Journal of Psychopathology and Behavioral Assessment, 23, 11-24. https:// doi.org/10.1023/A:1011035306061

Lilienfeld, S. O., \& Widows, M. R. (2005). Psychopathic Personality Inventory-Revised: Professional manual. Lutz, FL: Psychological Assessment Resources.

*Mager, K. L., Bresin, K., \& Verona, E. (2014). Gender, psychopathy factors, and intimate partner violence. Personality Disorders: Theory, Research, and Treatment, 5, 257267. https://doi.org/10.1037/per0000072

*Marshall, A. D., \& Holtzworth-Munroe, A. (2010). Recognition of wives' emotional expressions: A mechanism in the relationship between psychopathology and intimate partner violence perpetration. Journal of Family Psychology, 24, 21-30. https://doi. org/10.1037/a0017952

*Meehan, J. C., Holtzworth-Munroe, A., \& Herron, K. (2001). Maritally violent men's heart rate reactivity to marital interactions: $A$ failure to replicate the Gottman et al. (1995) typology. Journal of Family Psychology, 15, 394-408. https://doi.org/10.1037//08933200.15.3.394

Miller, J. D., Lamkin, J., Maples-Keller, J. L., \& 
Lynam, D. R. (2016). Viewing the triarchic model of psychopathy through general personality and expert-based lenses. Personality Disorders: Theory, Research, and Treatment, 7, 247-258. https://doi. org/10.1037/per0000155

Moher, D., Liberati, A., Tetzlaff, J., Altman, D. G., \& The PRISMA Group (2009). Preferred reporting items for systematic reviews and meta-analyses: The PRISMA statement. Annals of Internal Medicine, 151, 264-269. https://doi.org/10.7326/0003-4819-1514-200908180-00135

Moreira, D., Almeida, F., Pinto, M., \& Fávero, M. (2014). Psychopathy: A comprehensive review of its assessment and intervention. Aggression and Violent Behavior, 19, 191-195. https:// doi.org/10.1016/i.avb.2014.04.008

*Murphy, C. M., Taft, C. T., \& Eckhardt, C. I. (2007). Anger problem profiles among partner violent men: Differences in clinical presentation and treatment outcome. Journal of Counseling Psychology, 54, 189-200. https:// doi.org/10.1037/0022-0167.54.2.189

Myers, W. C., \& Monaco, L. (2000). Anger experience, styles of anger expression, sadistic personality disorder, and psychopathy in juvenile sexual homicide offenders. Journal of Forensic Sciences, 45, 698-701. https://doi. org/10.1520/JFS14753J

Neal, T. M., \& Sellbom, M. (2012). Examining the factor structure of the Hare Self-Report Psychopathy Scale. Journal of Personality Assessment, 94, 244-253. https://doi.org/1 $0.1080 / 00223891.2011 .648294$

*Okano, M., Langille, J., \& Walsh, Z. (2016). Psychopathy, alcohol use, and intimate partner violence: Evidence from two samples. Law and Human Behavior, 40, 517-523. https:// doi.org/10.1037//hb0000192

*Panuzio, J., O'Farrell, T., Marshall, A. D., Murphy, C. M., Murphy, M., \& Taft, C. T. (2006). Intimate partner aggression reporting concordance and correlates of agreement among men with alcohol use disorders and their female partners. Assessment, 13, 266-279. https:// doi.org/10.1177/1073191106287792

Paulhus, D., Neumann, C. S., \& Hare, R. D. (2016). The SRP-4: Self-Report Psychopathy Scale-Fourth Edition. Toronto, ON: MultiHealth Systems

Perestelo-Pérez, L. (2013). Standards on how to develop and report systematic reviews in Psychology and Health. International Journal of Clinical and Health Psychology, 13, 49-57. https://doi.org/10.1016/\$16972600(13)70007-3

Pérez, B., Herrero, J., Velasco, J., \& RodríguezDíaz, F. J. (2015). A contrastive analysis of the factorial structure of the PCL-R: Which model fits best the data? The European Journal of Psychology Applied to Legal Context, 7, 23-30. https://doi.org/10.1016/i. ejpal.2014.10.001

Pérez, B., Rodríguez-Díaz, F. J., Herrero, J., \& Fernández-Suárez, A. (2016). A profile of institutionalized psychopaths without a history of criminal behavior before incarceration. Terapia Psicológica, 34, 81-91. https://doi. org/10.4067/S0718-48082016000200001

Reidy, D. E., Kearns, M. C., \& DeGue, S. (2013). Reducing psychopathic violence: A review of the treatment literature. Aggression and Violent Behavior, 18, 527-538. https://doi. org/10.1016/i.avb.2013.07.008

Reidy, D. E., Kearns, M. C., DeGue, S., Lilienfeld, S. O., Massetti, G., \& Kiehl, K. A. (2015). Why psychopathy matters: Implications for public health and violence prevention. Aggression and Violent Behavior, 24, 214-225. https:// doi.org/10.1016/i.avb.2015.05.018

Richards, D. (2008). Handsearching still a valuable element of the systematic review. Evidence-Based Dentistry, 9, 85. https://doi. org/10.1038/si.ebd.6400602

Richards, H. J., Gacono, C. B., Cunliffe, T. B., Kivisto, A. J., Smith, J. M., \& Bodholdt, R. (2016). Assessing psychopathy in adults: The Hare Psychopathy Checklist-Revised and Psychopathy Checklist Screening Version. In C. B. Gacono (Ed.), The clinical and forensic assessment of psychopathy. A practitioner's guide (2 ed.) (pp.137-166). New York: Routledge.

Roche, M. J., Shoss, N. E., Pincus, A. L., \& Ménard, K. S. (2011). Psychopathy moderates the relationship between time in treatment and levels of empathy in incarcerated male sexual offenders. Sexual Abuse: A Journal of Research and Treatment, 23, 171-192. https://doi. org/10.1177/1079063211403161

*Rock, R. C., Sellbom, M., Ben-Porath, Y. S., \& Salekin, R. T. (2013). Concurrent and predictive validity of psychopathy in 
a batterers' intervention sample. Law and Human Behavior, 37, 145-154. https://doi. org/10.1037//hb0000006

Salekin, R. T. (2002). Psychopathy and therapeutic pessimism. Clinical lore or clinical reality? Clinical Psychology Review, 22, 79-112. https://doi.org/10.1016/S02727358(01)00083-6

Salekin, R. T., Worley, C., \& Grimes, R. D. (2010). Treatment of psychopathy: A review and brief introduction to the Mental Model Approach for psychopathy. Behavioral Sciences and the Law, 28, 235-266. https://doi.org/10.1002/ bsl.928

Salvador, B., Arce, R., Rodríguez-Díaz, F. J., \& Seijo, D. (2017). Psychometric assessment of psychopathy: A meta-analytical review. Revista Latinoamericana de Psicología, 49, 36-47. https://doi.org/10.1016/i.rlp.2015.09.015

Salvador, B., Pérez, B., Fernández, L., Bringas, C., \& Rodríguez-Díaz, F. J. (2015). La psicopatía: Una revisión bibliográfica y bibliométrica. Arquivos Brasileiros de Psicología, 67(2), 105-121. Retrieved from http://www.redalyc. org/articulo.oa? id =229042579009

Sawyer, S., Coles, J., Williams, A., \& Williams, B. (2015). Preventing and reducing the impacts of intimate partner violence: Opportunities for Australian ambulance service. Emergency Medicine Australasia, 27, 307-31 1. https:// doi.org/10.1111/1742-6723.12406

Scott, R. (2014). Psychopathy - An evolving and controversial construct. Psychiatry, Psychology, and Law, 21, 687-715. https://doi.org/10.10 80/13218719.2014.911056

Sitney, M. H., Caldwell, B. M., \& Caldwell, M. F. (2016). The longitudinal relationship between African American status, psychopathic traits, and violent recidivism in juvenile offenders. Criminal Justice and Behavior, 43, 1190-1203. https://doi. org/10.1177/0093854816645619

Siödin, A-K., Wallinius, M., Billstedt, E., Hofvander, B., \& Nilsson, T. (2017). Dating violence compared to other types of violence: Similar offenders but different victims. The European Journal of Psychology Applied to Legal Context, 9, 83-91. https://doi. org/10.1016/i.eipal.2017.03.001

Skeem, J., \& Cooke, D. (2010). Is criminal behavior a central component of psychopathy? Conceptual directions for resolving the debate. Psychological Assessment, 22, 433445. https://doi.org/10.1037/a0008512

Skeem, J. L., Poythress, N., Edens, J. F., Lilienfeld, S. O., \& Cale, E. M. (2003). Psychopathic personality or personalities? Exploring potential variants of psychopathy and their implications for risk assessment. Aggression and Violent Behavior, 8, 513-546. https:// doi.org/10.1016/S1359-1789/02)00098-8

*Stanford, M. S., Houston, R. J., \& Baldridge, R. M. (2008). Comparison of impulsive and premeditated perpetrators of intimate partner violence. Behavioral Sciences and the Law, 26, 709-722. https://doi.org/10.1002/bsl.808

Sturup, J., Karlberg, D., Fredriksson, B., Lihoff, T., \& Kristiansson, M. (2016). Risk assessments and recidivism among a population-based group of Swedish offenders sentenced to life in prison. Criminal Behaviour and Mental Health, 26, 124-135. https://doi.org/10.1002/ $\mathrm{cbm} .1941$

*Swogger, M. T., Walsh, Z., \& Kosson, D. S. (2007). Domestic violence and psychopathic traits: Distinguishing the antisocial batterer from other antisocial offenders. Aggressive Behavior, 33, 1-8. https://doi.org/10.1002/ ab.20185

*Swogger, M. T., Walsh, Z., Kosson, D. S., Cashman-Brown, S., \& Caine, E. D. (2012). Self-reported childhood physical abuse and perpetration of intimate partner violence: The moderating role of psychopathic traits. Criminal Justice and Behavior, 39, 910-922. https:// doi.org/10.1177/0093854812438160

${ }^{*}$ Taft, C. T., Murphy, C. M., Musser, P. H., \& Remington, N. A. (2004). Personality, interpersonal, and motivational predictors of the working alliance in group cognitivebehavioral therapy for partner violent men. Journal of Consulting and Clinical Psychology, 72, 349-354. https://doi. org/10.1037/0022-006X.72.2.349

*Theobald, D., Farrington, D. P., Coid, J. W., \& Piquero, A. R. (2015). Are male perpetrators of intimate partner violence different from convicted violent offenders? Examination of psychopathic traits and life success in males from community survey. Journal of Interpersonal Violence, 31, 1687-1718. https://doi. org/10.1177/0886260515569061

*Thornton, A. J. V., Graham-Kevan, N., \& Archer, J. (2016). Intimate partner violence: Are the 
risk factors similar for men and women, and similar to other types of offending? Aggressive Behavior, 42, 404-412. https:// doi.org/10.1002/ab.21635

Vassar, M., Atakpo, P., \& Kash, M. J. (2016). Manual search approaches used by systematic reviewes in dermatology. Journal of Medical Library Association, 104, 302-304. https:// doi.org/10.3163/1536-5050.104.4.009

*Walsh, Z., Swogger, M. T., O'Connor, B., Schonbrun, Y. C., Shea, M. T., \& Stuart, G. L. (2010). Subtypes of partner violence perpetrators among male and female psychiatric patients. Journal of Abnormal Psychology, 179, 563-574. https://doi. org/10.1037/a0019858
Wiklund, G., Ruchkin, V. V., Koposov, R. A., \& Af Klinteberg, B. (2014). Pro-bullying attitudes among incarcerated juvenile delinquents: Antisocial behavior, psychopathic tendencies and violent crime. International Journal of Law and Psychiatry, 37, 281-288. https://doi. org/10.1016/i.iilp.2013.11.019

*Wymbs, B. T., Dawson, A. E., Suhr, J. A., Bunford, N., \& Gidycz, C. A. (2017). ADHD symptoms as risk factors for intimate partner violence perpetration and victimization. Journal of Interpersonal Violence, 32, 659-681. https:// doi.org/10.1177/0886260515586371 